





\section{NBSIR 73.124 (R)}

\section{Evaluation of Currency and Stamp Papers}

E. L. Graminski and E. E. Toth

Paper Evaluation Section

Product Evaluation Technology Division

Institute for Applied Technology

January 30,1973

Progress Report covering the period

July 1 - December 31, 1972

\section{Prepared for}

Bureau of Engraving and Printing U.S. Department of the Treasury Washington, D. C. 20401 



\title{
EVALUATION OF CURRENCY AND STAMP PAPERS
}

\author{
E. L. Graminski and E. E. Toth
}

Paper Evaluation Section

Product Evaluation Technology Division

Institute for Applied Technology

January 30,1973

Progress Report covering the period

July 1 - December 31, 1972

Note

The results contained and the conclusions reached in this progress report are preliminary. Final results and conclusions will be presented in the final report.

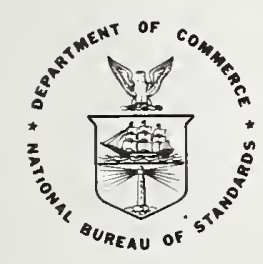

U. S. DEPARTMENT OF COMMERCE, Frederick B. Dent, Secretary

NATIONAL BUREAU OF STANDARDS, Richard W. Roberts, Director 

CONTENTS

$\underline{\text { Page }}$

1. SUMMARY .................. 1

2. EDGE TEAR OF REDEEMED CURRENCY . . . . . . . . . 3

3. MODIFICATION OF PAPER BY TREATMENT WITH ACRYLIC LATEXES • . . . . . . . . . . . . 5

3.1 Beater Addition of Acrylic Polymers . . . . 5

A. Experimental Details . . . . . . . . 5

B. Results and Discussion . . . . . . . 6

3.2 Paper Saturation with Acrylic Latexes . . . 8

A. Experimental Details . . . . . . . . 8

B. Results and Discussion . . . . . . . . 9

4. IREPARATION OF THE NIAGARA BEATER . . . . . . . . II

5. PLANS FOR FUTURE WORK . . . . . . . . . . . 12

6. PIBLIOGRAPHY . . . . . . . . . . . . . 13 



\section{SUMMARY}

As part of a continuing study for the Bureau of Engraving and Printing of the U.S. Department of Treasury, the disparity in the edge tear* of flexed currency paper and redeemed currency and the possibility of improving the stiffness retention of paper by treatment with acrylic resins was studied.

The edge tear of currency increases substantially as the condition of a note deteriorates during circulation. Conversely, the edge tear of currency paper increases only slightly at first then decreases during laboratory flexing. An investigation was conducted to determine if this disparity in edge tear behavior was due to significant differences in the structural changes of currency paper occurring during flexing and circulation or whether the disparity was due to the sampling procedure used to test redeemed currency and flexed currency paper. An investigation also was conducteó to determine whether modification of paper with acrylic latexes could improve the stiffness retention of paper and whether the method of modification affected the results.

In determining the edge tear of redeemed currency, the actual edges of notes are tested. In determining the edge tear of flexed currency paper, the edges of the flexed paper are purnosely avoided. The edge tear specimens of redeemed currency were retested on the edge opposite to the first test. This simulated sampling for edge tear testing of laboratory flexed paper since the edge used in the retest came from the interior of the note.

The edge tear of redeemed currency in the interior of a note is significantly lower than on the actual edge of the note and is essentially the same as it is for moderately flexed currency paper. Apparently, the increase in edge tear of currency during circulation is caused by edge wear and is not due to the flexing a note receives during circulation. These results further indicate that there is good agreement in the changes that occur in the properties of currency paper during currency circulation and laboratory flexing.

*Edge tear is the resistance offered by paper to the onset of tearing at the edge of a sheet. 
Handsheets were modified with acrylic polymers by beater addition and paper saturation techniques. The effect of polymer concentration on the extent of change in paper properties was also investigated. The handsheets were prepared from a kraft wood pulp beaten in a PFI laboratory mill.

The investigation of acrylic latexes demonstrated that modification of paper with acrylics can result in marked improvement in stiffness retention with flexing and that the best results are obtained when the modification is produced by paper saturation. There is a possibility that wood pulp paper, modified with polymer latexes, could be superior to currency paper manufactured from rag pulps.

The effect of acrylic polymer modification of paper on the retention of cantilever stiffness during flexing will be continued during the next reporting period. 


\section{EDGE TEAR OF REDEEMED CURRENCY}

The edge tear of redeemed currency is appreciably higher than that of uncirculated currency [1]. The edge tear of printed currency paper increases during the early stages of flexing but then decreases as flexing continues [1]. In any event, the average edge tear of flexed printed currency paper is never as high as the edge tear of redeemed currency. The difference in edge tear between flexed currency paper and redeemed currency may be due to significant differences in the structural changes of paper occurring during flexing and currency circulation.

In determining the edge tear of currency, care was taken in cutting the specimens to include the actual edge of the redeemed note [2]. In obtaining specimens for testing from flexed currency paper, care is taken not to include the actual edge of the flexed sample so as to avoid edge effects in testing. Therefore, the disparity in edge tear between redeemed currency and flexed currency paper could also be due to sampling procedures.

In determining the edge tear of redeemed currency or flexed currency paper, only one edge is tested per specimen and not both edges as suggested in TAPPI Method T 470 os-66. Photographs of the edge tear device used at NBS are shown in Figure 3 .

The same specimens used in determining the edge tear of redeemed currency [2] were tested a second time but on the opposite edge. This edge came from the interior of the note and would be free of edge effects. The second edge tear determination was made in the area directly opposite the first determination as shown in Figure I. The results are given in Table 1 .

The edge tear of redeemed currency is significantly lower in the interior of the note than on the actual edge of the note. However, it could be argued that the edge tear should be lower by virtue of the specimens being subjected to tensile forces during the first edge tear test. Therefore, the remainder of the untested portion of each redeemed bill was cut (as shown in Figure 1) into two specimens for additional edge tear testing. The previously untested specimens were tested on the side adjacent to the outer specimens and in approximately the same area (see Figure 1). The results are given in Table 1 under the column heading, Top and/or Eottom Control. 
The average edge tear for the control specimens was the same as the average for the original specimens tested on the side from the interior of the note. This indicates that the first edge tear test had no effect on the edge tear of the opposite side of the specimen.

The average edge tear of the interior of redeemed currency j.s essentially the same as it is for uncirculated currency. The higher edge tear for the actual edge of redeemed currency is apparently due to the wear such as abrasion, etc., received during circulation and not due to the flexing it receives. The edge tear data from the interior of redeemed currency are also in good agreement with the edge tear data obtained from flexed, printed currency paper, giving further indication that laboratory flexing is an excellent method for evaluating the durability of paper. 


\section{MODIFICATION OF PAPER BY TREATMENT WITH ACRYIIC LATEXES}

There are two methods for modifying paper with polymer latexes. One is the so-called beater addition which actually does not take place in a beater but in a mixing chest where the beaten pulp can be agitated gently in the presence of a latex. The second method is called paper saturation which involves saturating dry paper with a latex, squeezing out the excess, followed by drying. In beater addition, the fibers are completely coated with polymer prior to sheet formation. In paper saturation, the polymer is deposited only on tre exposed portion of the fibers. It is apparent that the effect of polymer on the physical properties of paper will be dependent on the method of polymer application to the paper. The object of this investigation was to determine the effect of acrylic polymers on the retention of stiffness when applied to paper by beater addition and paper saturation.

Four acrylic latexes (designated E-631, P-339, E-610, and $A C-61)$ were chosen for this investigation. The polymer stiffness was estimated by the manufacturer from the torsional modulus of an air-dried film. E-631 was the softest polymer, P-339 and E-610 were intermediate, and AC-61 was the stiffest of the four polymers evaluated.

\subsection{Beater Addition of Acry.lic Polymers}

A. Experimental Details

A bleached kraft wood pulp was beaten in a PFI laboratory mill at a 10 percent consistency with no clearance between bedplate and roll for 5,000 revolutions at $3.4 \mathrm{kilograms}$ force and a relative velocity of roll to bedplate of $6 \mathrm{~m} / \mathrm{sec}$. The beating was done in distilled water. Aliquots of this pulp, sufficient to make a $12^{\prime \prime} \times 12^{\prime \prime}$ handsheet of $70 \mathrm{~g} / \mathrm{m}^{2}$ basis weight, were diluted with 1.5 liters of distilled water and disintegrated for 7,500 revolutions in a British disintegrator. The $\mathrm{pH}$ is adjusted to $\mathrm{pH} 9$ with $1 \mathrm{~N} \mathrm{NaOH}$. A retention aid is added to the pulp slurry in the amount of 2 percent based on latex solids to be deposited on the fibers. The retention aid is added from a sufficient quantity of a 1 percent solution, diluted with $50 \mathrm{~cm}^{3}$ of distilled water. OnIy two-thirds of the retention aid was added at the start. The mixture of pulp suspension and retention aid was stirred 5 minutes prior to latex addition to exhaust the retention aid from solution. The $\mathrm{pH}$ of the mixture was then decreased to 4.0 with $0.5 \mathrm{~N} \mathrm{H}_{2} \mathrm{SO}_{4}$. 
The acrylic emulsion was diluted with approximately $50 \mathrm{~cm}^{3}$ distilled water and added to the pulp suspension in three equal portions with moderate stirring. Five minutes was allowed between each addition to exhaust the acrylic latex. Only moderate stirring was used in order not to remove any adsorbed polymer by shearing. After all of the latex was added, the remainder of retention aid was added and the mixture stirred for an additional 5 minutes. Handsheets were then prepared by placing the mixture in the deckle box of the handsheet machine and forming the sheet in the usual way using tap water. The sheets were dried at $95^{\circ} \mathrm{C}$ for approximately 3 minutes on a drum dryer.

The effect of the acrylics on the retention of cantilever stiffness was evaluated by determining the decline in cantilever stiffness after 1,000 double flexes over 1/8" rollers on the NBS paper flexer. All of the tensile properties and other physical properties were determined in addition to the cantilever stiffness. The results are given in Tables 2-5 and the standard deviation of the results are given in Tables 6-9.

\section{B. Results and Discussion}

The extensional stiffness of the handsheets decreased as the aniount of polymer deposited on the fibers increased. The decrease in extensional stiffness was greatest with E-631, the softest acrylic evaluated, and least with AC-61, the stiffest of, the four acrylics. Breaking strength was affected most by the stiffest polymer (AC-6I), while improvement in elongation to break was greatest with $\mathrm{P}-339$, an acrylic with moderate stiffness. The effect of acrylic polymer on the energy to break, yield load, and elongation to break was not very great. Plastic stiffness decreased extensively with handsheets containing E-631, while the remaining three polymers had somewhat less effect on this property.

None of the acrylic polymers evaluated appeared to have any great effect on the initial cantilever stiffness of paper. Their effect on folding endurance ranged from a significant decrease with E-63I (two sided t-test at 95 percent confidence interval) to a significant increase with $\mathrm{P}-339$ and E-610. With the exception of AC-61, each of the acrylic latexes caused the air permeability to increase with increasing amounts of polymer treatment. 
Of the tensile properties, only initial stiffness and elongation to yield are affected substantially after 1,000 flexes. There is a large decrease in the initial stiffness and a large increase in elongation to yield. Small but significant increases in elongation to break occurred with all handsheets investigated. The remaining tensile properties exhibited little or no change after 1,000 flexes.

Cantilever stiffness declined extensively after 1,000 flexes. The only real significant improvement in stiffness retention over the controls occurred with handsheets containing 5 and 10 percent $A C-61$. However, the improvement in stiffness retention was not as great as observed in handsheets treated with wet strength resin.

It is apparent from the above results that none of the acrylics evaluated when deposited in paper by beater addition improve stiffness retention of papers adequately. 


\subsection{Paper Saturation with Acrylic Latexes}

A. Experimental Details

The same wood pulp was used in this investigation as was used in the beater addition investigation, and the beating was done as described in section 3.1A. A total of $600 \mathrm{~g}$ pulp was beaten in 15 separate charges then combined in a large stainless steel container. The pulp was diluted with sufficient distilled water to make a 1 percent suspension and was stirred vigorously for 1 hour prior to handsheet preparation. Aliquots of the 1 percent suspension were treated in the British disintegrator for 7,500 revolutions, transferred to the deckle box of the handsheet mold, and sheets were made in the usual way. Each sheet was weighed after drying and only those sheets whose basis weight was $70 \mathrm{~g} / \mathrm{m}^{2} \pm 5$ percent were retained. The sheets were then separate $\bar{d}$ into ten groups of six sheets each by a random selection.

The paper saturation was performed as follows: The felts which are used in wet pressing of handsheets were saturated with either a 5 or 10 percent emulsion of the acrylic. A handsheet was placed in the felt and passed through the calender rolls on the sheet machine. As the felt passed through the calendar rolls, the excess latex was squeezed out, saturating the paper with latex. As the felt and paper proceeded through the rolls, the excess latex in the paper was squeezed out. The wet sheet saturated with acrylic was lifted from the felt and dried on the drying drum at $95^{\circ} \mathrm{C}$ for approximately 3 minutes. The weight of the sheet was determined after drying and by difference, the percent of polymer in the sheet was determined.

The effect of acrylic on the retention of physical properties was evaluated by determining the decline in physical properties after 1,000 double flexes over $1 / 8$ inch rollers on the NBS paper flexer. The results are given in Tables 10-13, and the standard deviation of the results are given in Tables 14-17. 
B. Results and Discussion

It is quite apparent from the results that more and greater changes occurred in tensile properties when the handsheets were modified with acrylics by saturation than by beater addition. Extensional stiffness, breaking strength, elongation to break, energy to break, and plastic stiffness all exhibited significant increases (two sided t-test) over the controls for all of the latexes except E-631. Overall, the greatest increase in tensile properties occurred with sheets modified with $\mathrm{AC}-6 \mathrm{I}$.

With the exception of sheets modified with E-63I, Elmendorf tear decreased as a result of saturation with acrylic latexes. Fold endurance either remained essentially unchanged or increased, and in practically every case, air permeability decreased after modification. Cantilever stiffness decreased in all. instances except for sheets containing AC-öl.

The decline in extensional stiffness with flexing is less with the handsheets modified by the saturation technique than with those treated by beater addition, while the increase in elongation to yield is lower for the saturated sheets. The only other tensile property exhibiting a significant change after flexing is the increase in elongation to break. All other tensile properties were virtually unchanged after 1,000 flexes.

Of greatest importance is the retention of stiffness of the sheets modified by saturation. Stiffness retention for all the modified handsheets was greater than the controls as shown in Figure 2. There is no doubt about the superiority of paper saturation over beater addition with respect to stiffness retention. In fact, stiffness retention was greater for handsheets modified with 8.5 percent AC-61 than for any paper evaluated to date, which includes currency paper. This is significant, as the pulp used in this evaluation was a wood pulp and not a rag pulp. Rag pulp is considerably more expensive and considered to be superior to wood pulp.

These investigations indicate that paper can be modified with acrylic resins resulting in a significant improvement in cantilever stiffness retention. The magnitude of the improvement in stiffness retention apparently depends to 
a degree on the rheology of the polymer as indicated by the results and quite probably on paper structure and pulp fiber rheology. The great improvement in stiffness retention of a wood pulp paper might result in a superior currency paper which is less expensive than currency paper made from rag pulps. 


\section{PREPARATION OF THE NIAGARA BEATER}

The piagara beater closely resembles the type of beater generally used in rag paper manufacture. It enables rag pulp to be fibrillated extensively without reduction of fiber length which up to now has been an important requirement for currency paper and for durable papers in general.

A newly acquired Niagara beater must first be "ground in" (a time-consuming operation) before reproducible results can be obtained. During this reporting period, the beater was "ground in," and it is anticipated that a schedule for beating cotton and linen will be worked out during the next reporting period. 
5. PIANS FOR FUTURE WORK

1. Continue the investigation on the improvement of stiffness retention by paper saturation of polymeric latexes.

2. Develop beater schedules for rag pulps on the Niagara beater for producing a currency type paper in the laboratory.

\section{BIBLIOGRAPHY}

1. Graminski, E. I. and Toth, E. E., NBS Report 10 090, Evaluation of Currency and Stamp Papers.

2. Graminski, E. I. and Forshee, B. W., NBS Report 9597, Evaluation of Currency and Stamp Papers, July 31, 1967.

3. Wilson, W. K. and Forshee, B. W. NBS Report 7198, Evaluation of Currency and Stamp Papers, July 15, 1961. 
Table 1. Edge tear of redeemed currency at various positions in a note.

\begin{tabular}{|c|c|c|c|c|c|c|}
\hline $\begin{array}{l}\text { Serial No. } \\
\text { of Note } \\
\end{array}$ & $\begin{array}{l}\text { Top } \\
\text { Edge } \\
\end{array}$ & $\begin{array}{c}\text { Top } \\
\text { Interior } \\
\text { Edge } \\
\end{array}$ & $\begin{array}{c}\text { Top } \\
\text { Control } \\
\end{array}$ & $\begin{array}{c}\text { Bottom } \\
\text { Edge }\end{array}$ & $\begin{array}{c}\text { Bottom } \\
\text { Interior } \\
\text { Edge } \\
\end{array}$ & $\begin{array}{l}\text { Bottom } \\
\text { Control } \\
\end{array}$ \\
\hline & & & force, & ilograms & & \\
\hline $\mathrm{Cl} 3322220 \mathrm{~A}$ & 1.43 & 0.95 & 0.52 & 0.93 & 0.68 & 0.50 \\
\hline E48965305B & 0.85 & 1.09 & 1.15 & 2.03 & 0.74 & 0.33 \\
\hline L70 $573237 B$ & 0.80 & 0.36 & 0.84 & 1.16 & 0.62 & 0.81 \\
\hline F03214779A & 1.04 & 0.72 & 0.56 & 1.10 & 0.69 & 0.43 \\
\hline B44442898A & 0.56 & 0.58 & 0.58 & 0.71 & 0.68 & 0.82 \\
\hline C12248091A & 1.34 & 0.90 & 1.46 & 1.40 & 0.87 & 0.50 \\
\hline E54593181B & 1.92 & 0.68 & 0.60 & 1.36 & 0.65 & 0.58 \\
\hline F87500623B & 0.89 & 0.89 & 0.59 & 1.12 & 0.53 & 1.01 \\
\hline A $51449725 \mathrm{~A}$ & 0.60 & 0.66 & 0.46 & 0.92 & 0.66 & 0.93 \\
\hline I01112392A & 1.93 & 1.01 & 0.81 & 0.80 & 1.14 & 0.69 \\
\hline B45657992A & 1.34 & 0.56 & 0.84 & 0.52 & 0.38 & 0.66 \\
\hline E30313683A & 1.81 & 0.46 & 0.65 & 1.18 & 0.66 & 0.85 \\
\hline$E 27910176 \mathrm{~A}$ & 1.62 & 1.49 & 0.79 & 0.68 & 1.05 & 1.36 \\
\hline B53207398B & 1.19 & 0.83 & 0.73 & 0.60 & 0.62 & 0.53 \\
\hline $\mathrm{E} 49645561 \mathrm{~B}$ & 0.92 & 0.92 & 0.66 & 0.54 & 0.61 & 0.97 \\
\hline $\mathrm{H} 62119580 \mathrm{~A}$ & 0.90 & 0.68 & 0.57 & 1.05 & 0.34 & 0.61 \\
\hline $\mathrm{F} 62862627 \mathrm{~A}$ & 1.22 & 0.73 & 0.92 & 1.48 & 1.12 & 0.92 \\
\hline J $21513136 \mathrm{~A}$ & 0.76 & 0.39 & 0.65 & 0.85 & 0.89 & 0.36 \\
\hline LII257762B & 0.77 & 0.58 & 0.69 & 1.58 & 1.82 & 0.78 \\
\hline B21823744A & 2.00 & 0.47 & 0.85 & 0.84 & 0.66 & 0.95 \\
\hline Average & 1.19 & 0.75 & 0.75 & 1.04 & 0.77 & 0.71 \\
\hline Std. Dev. & 0.46 & 0.27 & 0.23 & 0.39 & 0.33 & 0.25 \\
\hline $\begin{array}{l}\text { 95\% confi- } \\
\text { dence in- } \\
\text { terval for } \\
\text { true mean }\end{array}$ & $1.19 \pm .22$ & $0.75 \pm .13$ & $0.75 \pm .11$ & $1.04 \pm .18$ & $0.77 \pm .15$ & $0.71 \pm .12$ \\
\hline
\end{tabular}




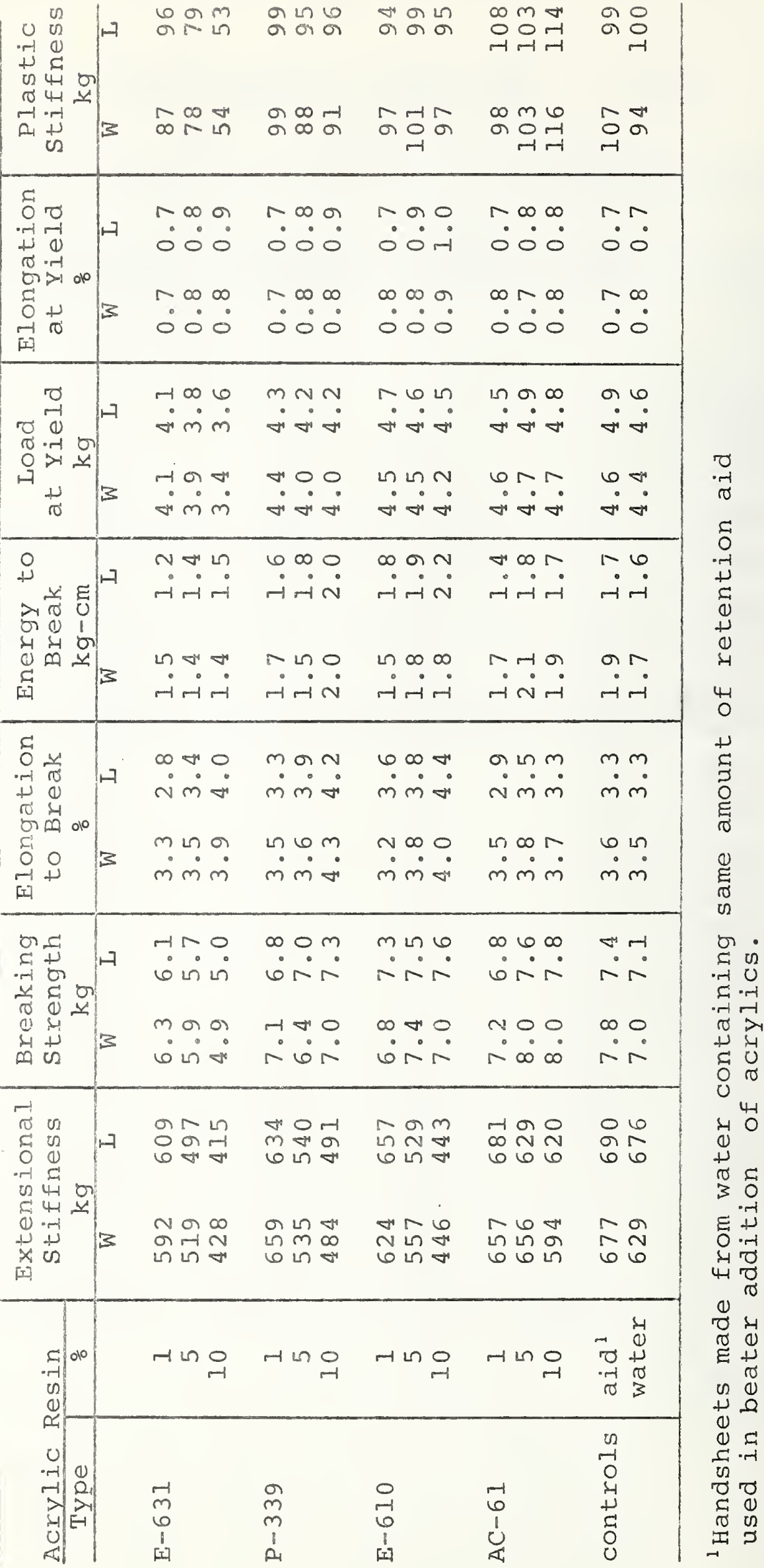




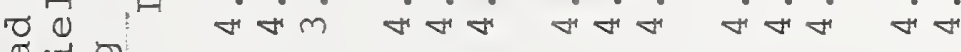

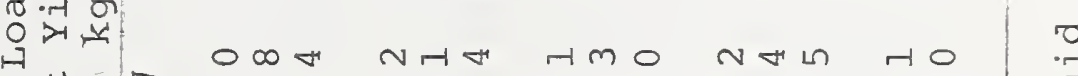

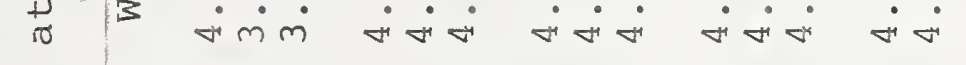

弄

خुळ

(1)

⿷匚

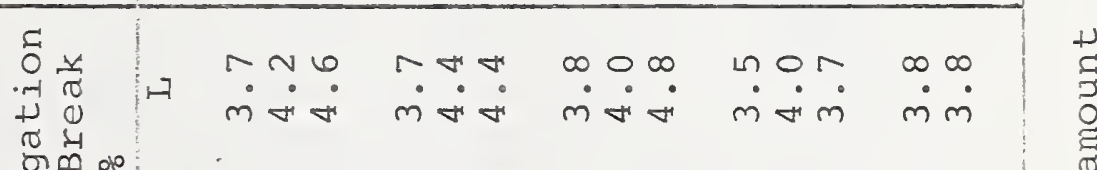

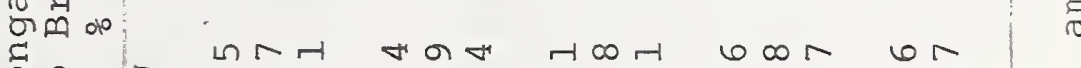

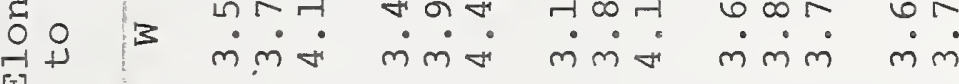

[T]

$\checkmark$

(1) $>$.

- 1 - 2

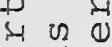

(1) $2 \pi$

ח.न-

- U 0

पy 41

다 4

(1) $0 \frac{5}{0}$

न-A

H

$\Omega>$

(1)

E-

$m$

Gू तर

. on

들

ग)

मी

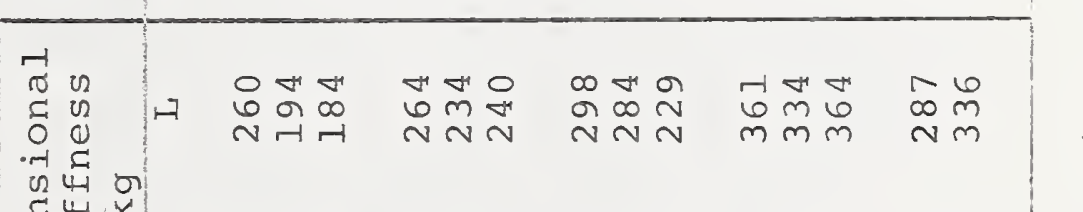

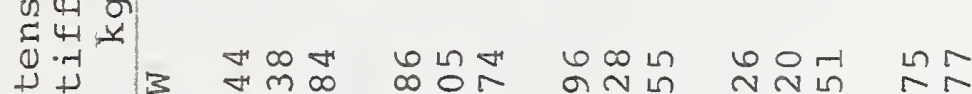

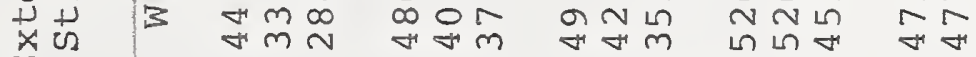
䦿

$\frac{0}{2}$

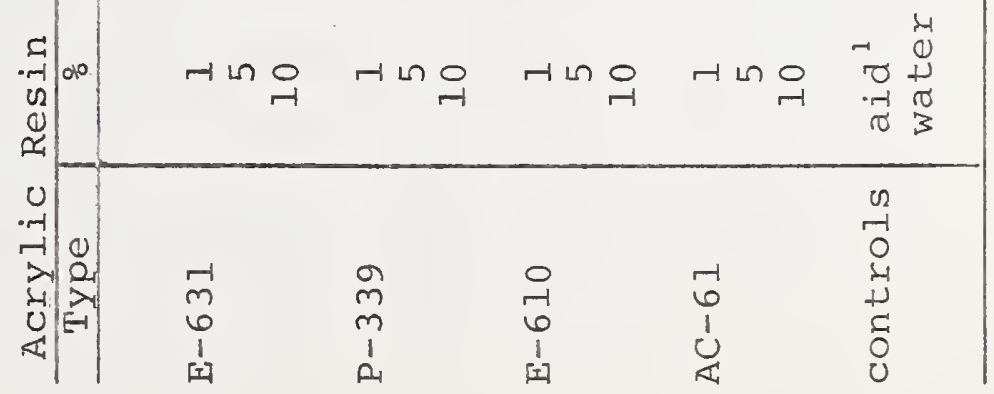




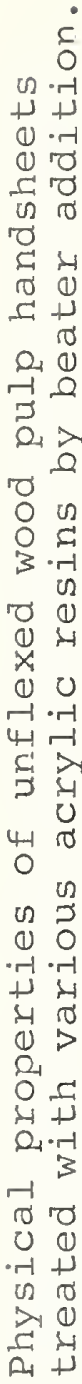

\begin{tabular}{|c|c|c|c|c|c|c|}
\hline 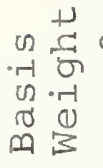 & $\frac{v_{\sigma}}{\xi}$ & $\begin{array}{l}n \\
0 N \infty\end{array}$ & $\begin{array}{l}0 \\
N\end{array}$ & $\begin{array}{c}\text { ON } \\
0\end{array}$ & $\stackrel{\circ}{N}$ & rio \\
\hline 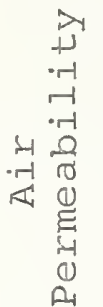 & $\begin{array}{l}N_{E}^{E} \\
U \\
U\end{array}$ & 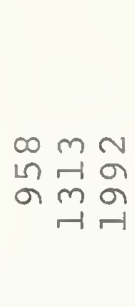 & 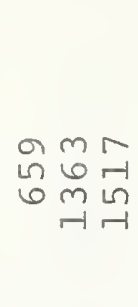 & $\begin{array}{lll}0 & \infty & 0 \\
0 & \kappa & 0 \\
\infty & \sigma & m \\
& \end{array}$ & 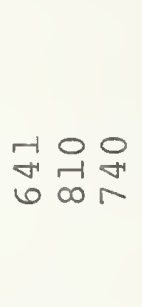 & $\begin{array}{ll}6 & 6 \\
\ddots & 0 \\
\infty & 6\end{array}$ \\
\hline
\end{tabular}

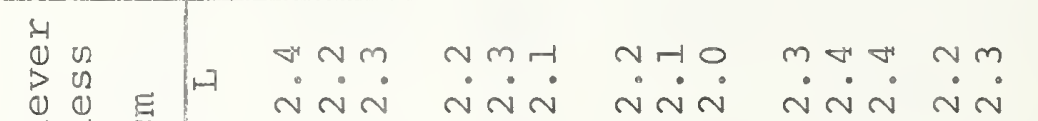

(1) $E$ NNN NNN NNN NNN N

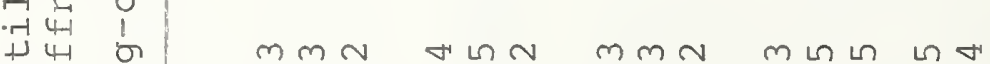

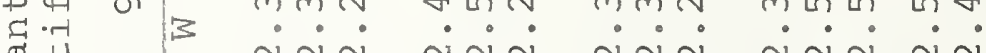

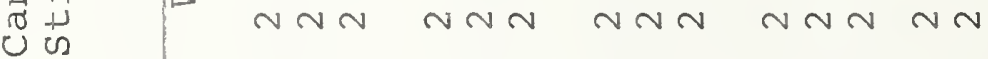

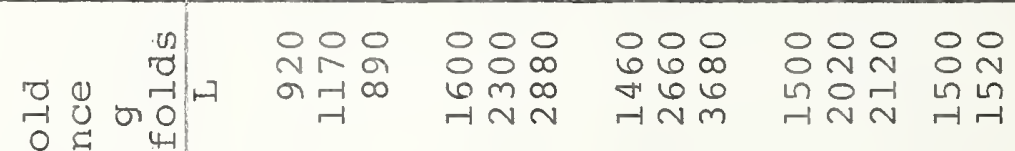

[ᄃ

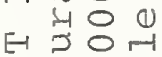

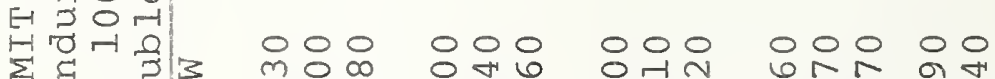

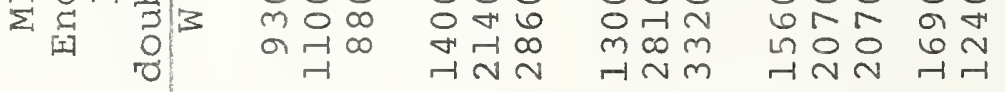

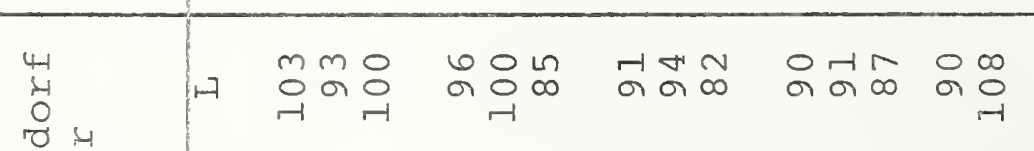

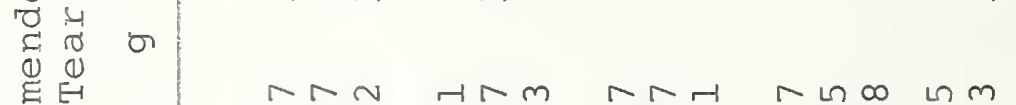

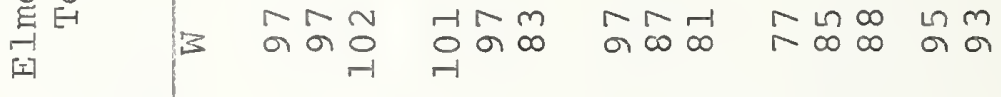

m

$\Rightarrow$ य

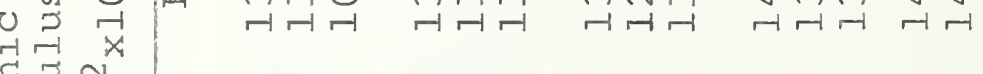

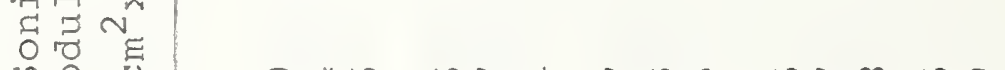

थ 0 U 0 \%

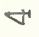

常

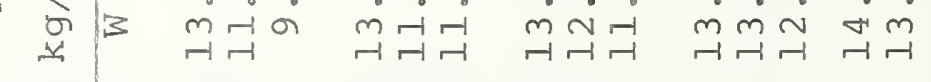

\begin{tabular}{|c|c|c|c|c|c|c|}
\hline $\begin{array}{c}5 \\
-1 \\
0 \\
0 \\
04\end{array}$ & 010 & r & rl 1 ( & 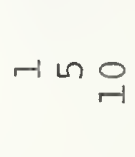 & ri L & $\begin{array}{cc} & y \\
m & 0 \\
\sigma & \pm \\
\cdot-1 & \sigma \\
\sigma & 3\end{array}$ \\
\hline $\begin{array}{c}0 \\
0-1 \\
-1 \\
\lambda \\
4 \\
0 \\
4\end{array}$ & 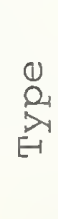 & $\begin{array}{c}-1 \\
n \\
0 \\
1 \\
\text { r. }\end{array}$ & $\begin{array}{l}0 \\
m \\
m \\
1 \\
n_{1}\end{array}$ & $\begin{array}{l}0 \\
-1 \\
6 \\
1 \\
\text { la }\end{array}$ & $\begin{array}{l}01 \\
\vdots \\
0 \\
1\end{array}$ & 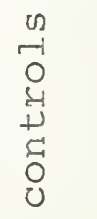 \\
\hline
\end{tabular}

$\therefore$.

in -4

- $\mathrm{r}$ \%

त)

崰

C

गैद ध

- $\frac{1}{3}$

(

ฮิं है

(1) -1

o

04

ᄀु 0

त 6

तथ

0 .

5

वह क्ष

ข

\%

(1) 实

4

(1)

3 U

in UN 4

¿ 0 E 0

${ }_{-\rightarrow 1}^{0}$ 皮

मम

न

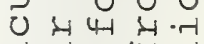

ना पन

ช

य $04 \pi$ T०

ग 0 E

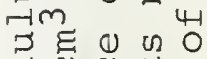

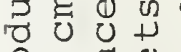

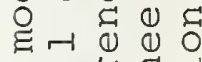

O 11 प्र

- -1 प

•

О

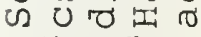




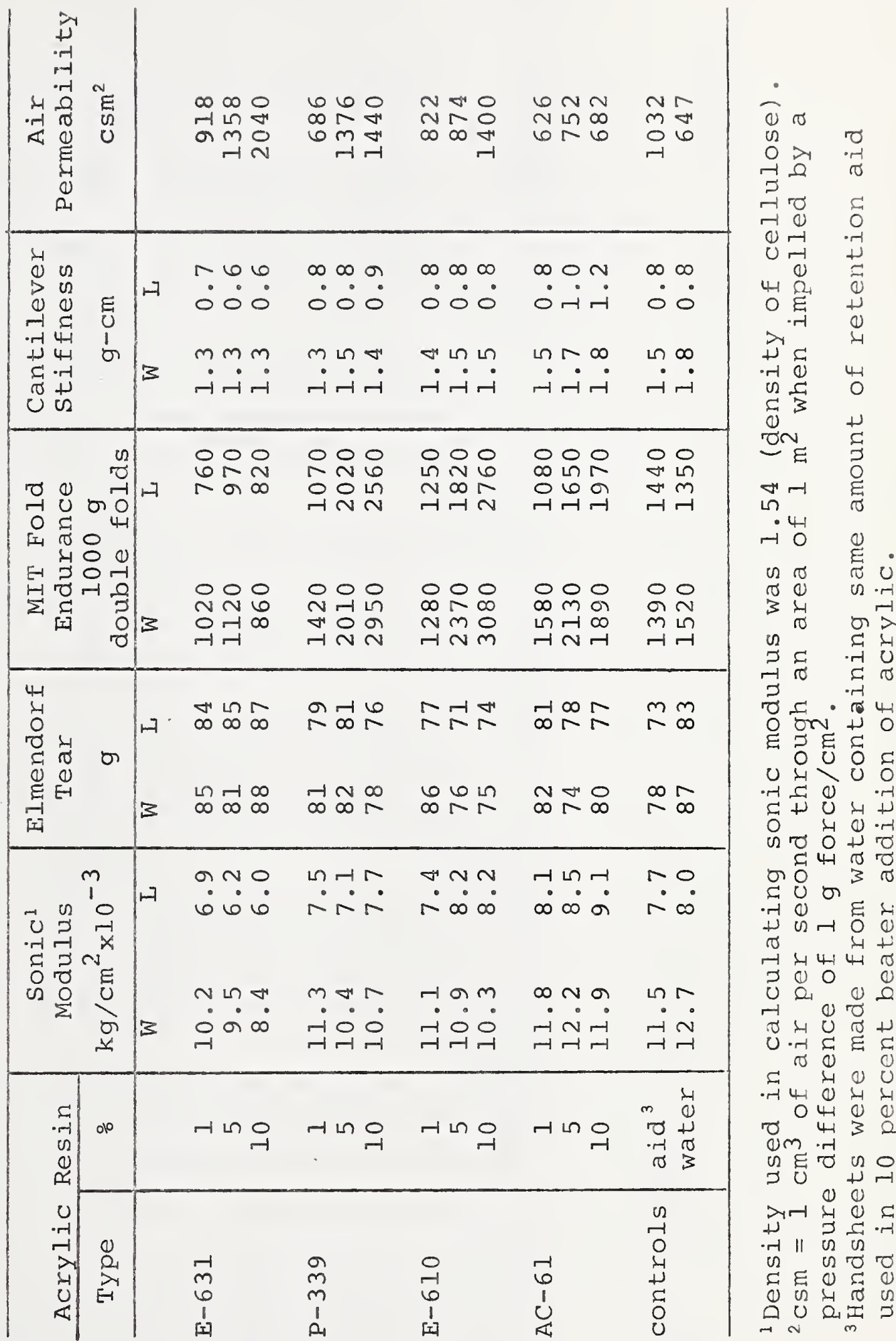




\begin{tabular}{|c|c|c|c|c|c|c|}
\hline 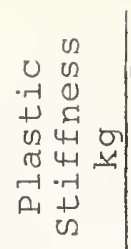 & 1 & 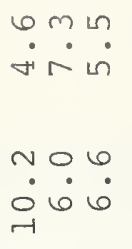 & 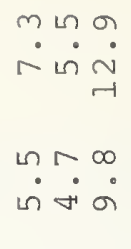 & 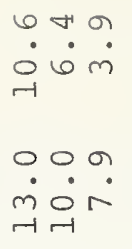 & 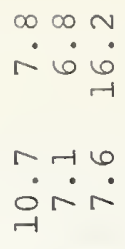 & 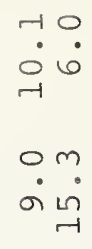 \\
\hline 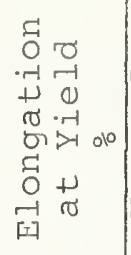 & 3 & 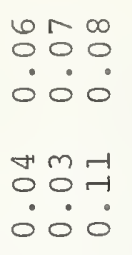 & 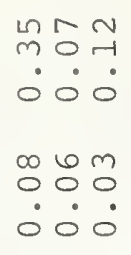 & 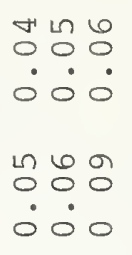 & 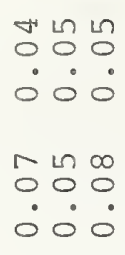 & 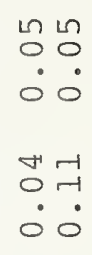 \\
\hline 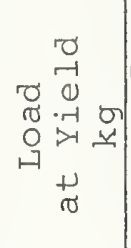 & $\mapsto$ & 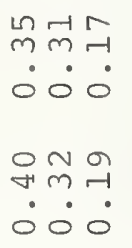 & 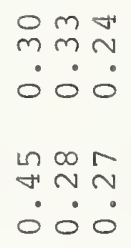 & 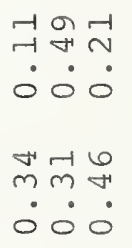 & 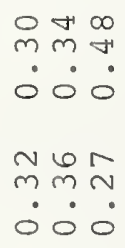 & 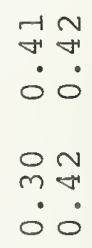 \\
\hline 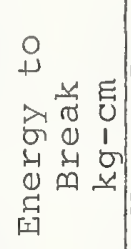 & 1 & 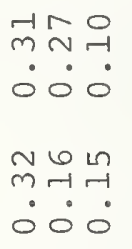 & 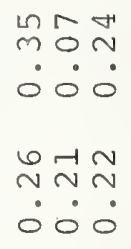 & 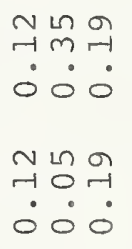 & 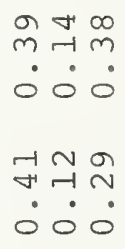 & $\begin{array}{l}\stackrel{H}{\circ} \stackrel{ }{m} \\
\dot{0} 0 \\
m \stackrel{n}{N} \\
\stackrel{0}{0}\end{array}$ \\
\hline 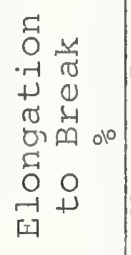 & 3 & 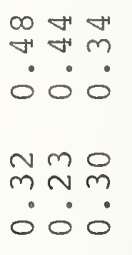 & 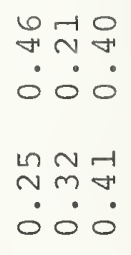 & 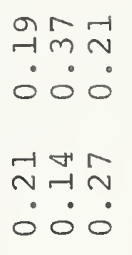 & 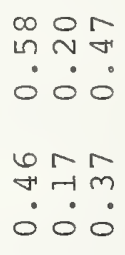 & 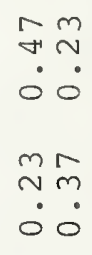 \\
\hline 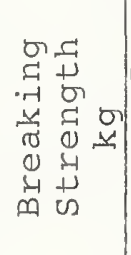 & $\mapsto$ & 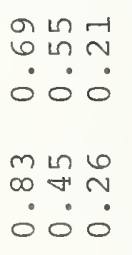 & 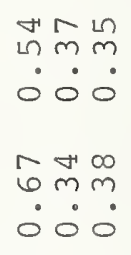 & 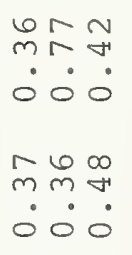 & 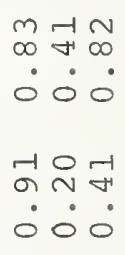 & 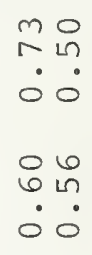 \\
\hline 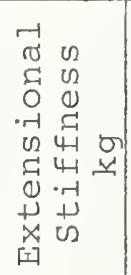 & $\mapsto$ & 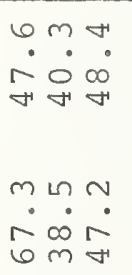 & $\begin{array}{lll}0 & 0 & 0 \\
ن & 0 & 0 \\
\sim & 0 & m\end{array}$ & 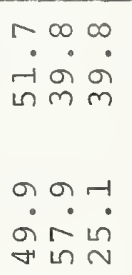 & 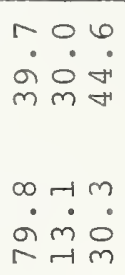 & 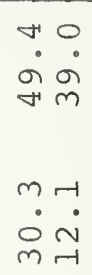 \\
\hline 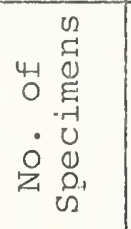 & $\mapsto$ & $\bullet \bullet \bullet$ & $6 \bullet$ & ! 6 & 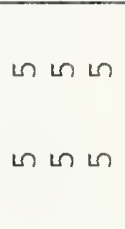 & $6 \Omega$ \\
\hline 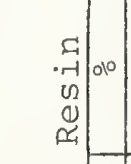 & & ની & r & 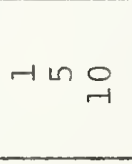 & $\rightarrow$ in $\underset{r}{0}$ & 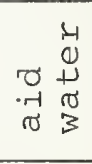 \\
\hline 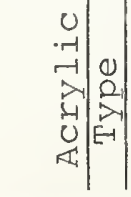 & & $\begin{array}{l}-1 \\
m \\
0 \\
1 \\
\text { |1 }\end{array}$ & $\begin{array}{l}a \\
m \\
m \\
1 \\
n_{1}\end{array}$ & $\begin{array}{l}0 \\
-1 \\
6 \\
1 \\
1\end{array}$ & \begin{tabular}{l}
-1 \\
6 \\
1 \\
0 \\
\multirow{4}{4}{}
\end{tabular} & $\begin{array}{l}0 \\
+1 \\
0 \\
0 \\
4 \\
1 \\
5 \\
0 \\
0\end{array}$ \\
\hline
\end{tabular}




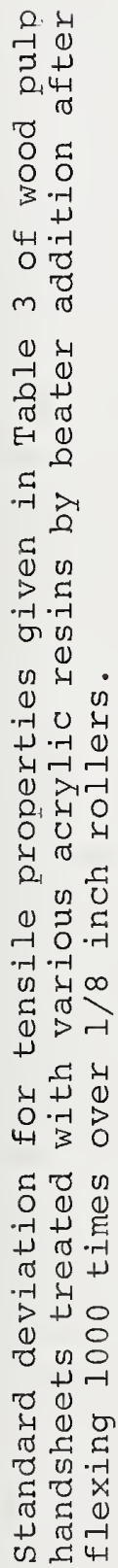

\begin{tabular}{|c|c|c|c|c|c|c|c|}
\hline 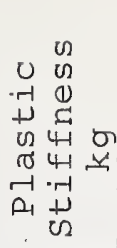 & $\mapsto$ & $\begin{array}{c}m \sim \dot{H} \\
\dot{0} \dot{+} \\
\dot{m} \dot{m} \\
\dot{\infty} \dot{0} \dot{\nabla}\end{array}$ & 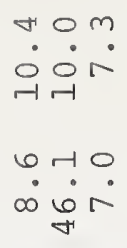 & 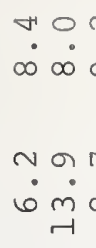 & & $\begin{array}{l}m \sim c \\
\dot{-1} \dot{r} \\
m o r \\
\dot{r} \text { in }\end{array}$ & 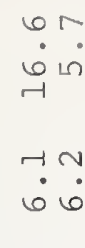 \\
\hline 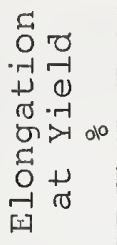 & 13 & 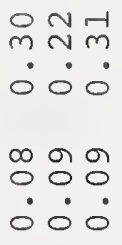 & 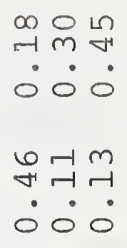 & 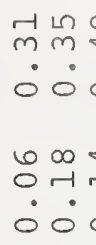 & $\begin{array}{l}\text { ब } \\
\dot{0}\end{array}$ & 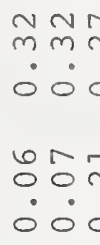 & $\begin{array}{l}N \text { m } \\
\dot{0} 0 \\
m 0 \\
-10 \\
00\end{array}$ \\
\hline 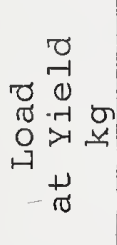 & 17 & 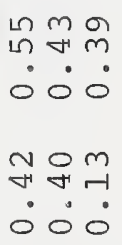 & 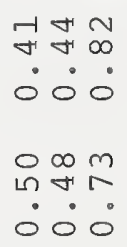 & 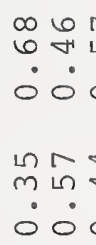 & in & 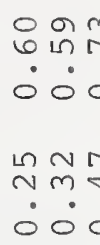 & 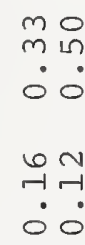 \\
\hline 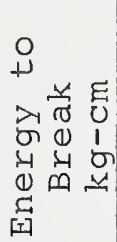 & $\mapsto$ & 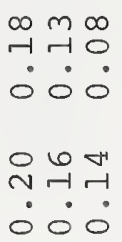 & 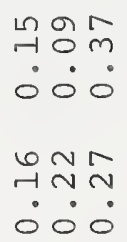 & 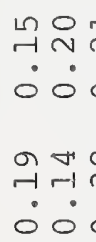 & ㅁ & 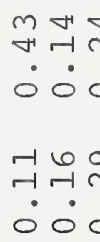 & 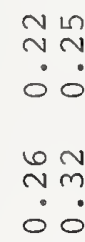 \\
\hline 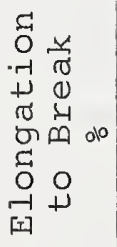 & 3 & 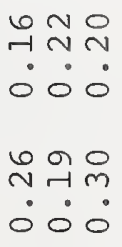 & 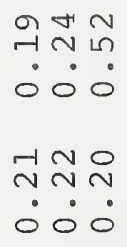 & 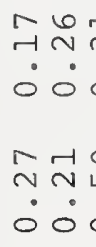 & m. & 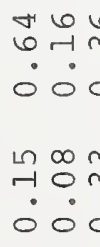 & $\begin{array}{l}\text { r m } \\
\dot{0} 0 \\
\text { m m } \\
\dot{0} 0\end{array}$ \\
\hline 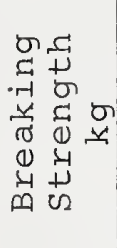 & $\mapsto$ & 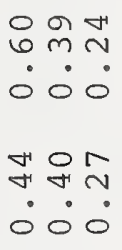 & 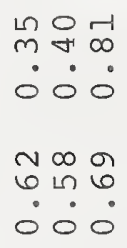 & 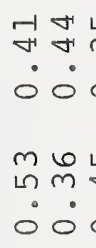 & $\dot{m}$ & 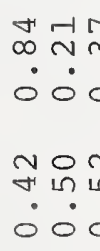 & $\begin{array}{l}m \infty \\
\text { in } \\
0 \\
0 \\
\text { म' }\end{array}$ \\
\hline 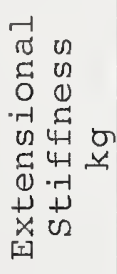 & $\mapsto$ & 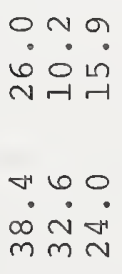 & 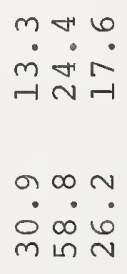 & 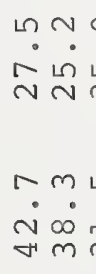 & $\begin{array}{l}0 \\
\dot{m}\end{array}$ & 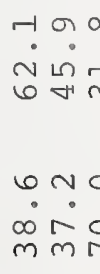 & 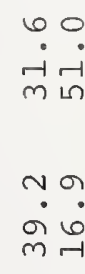 \\
\hline 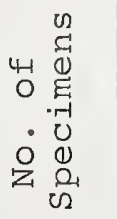 & 11 & ص 6. & 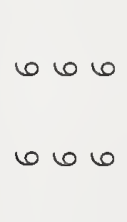 & 66 & 6 & $\begin{array}{l}\llcorner\cap \operatorname{LL} L \\
\operatorname{LL} L\end{array}$ & 6\llcorner \\
\hline 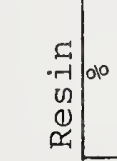 & & ન & r & $r$ L & $\underset{r-1}{0}$ & -1 เ & $\begin{array}{c}4 \\
0 \\
0 \\
0 \\
-7 \\
\pi \\
\pi \\
3\end{array}$ \\
\hline 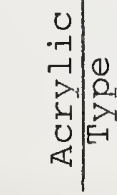 & & $\begin{array}{l}-1 \\
m \\
0 \\
1 \\
{[-1}\end{array}$ & $\begin{array}{c}a \\
m \\
m \\
1 \\
n\end{array}$ & $\begin{array}{l}0 \\
-1 \\
6 \\
1 \\
\text { 되 }\end{array}$ & & \begin{tabular}{l}
-1 \\
0 \\
1 \\
$u$ \\
\multirow{1}{1}{}
\end{tabular} & 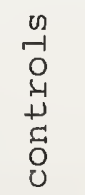 \\
\hline
\end{tabular}


E.

\begin{tabular}{l}
10 \\
0 \\
\hline 1
\end{tabular}

$>+\infty$

on 0.

u

0 \%

स

(4)

(1) 04

요묘

$4 \pi 0$

ㅇ. Б0

-1

ठ

- 1 त्र

ज) थ

고

है

00

40

4

त् .

ᄃ $x$

- -1 (1)

\-1 0

( $4 \pi$

$\rightarrow 5$

82

ठำ

$\nabla$

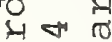

$\sigma>$

त 0

б요엄

$+\sigma \cdot-1$

OS $\mathrm{EH} 3$

$\infty$
0
0
0
0

\begin{tabular}{|c|c|c|c|c|c|c|c|c|}
\hline$\cdot 4$ & 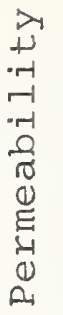 & $\begin{array}{l}E \\
u \\
u\end{array}$ & & 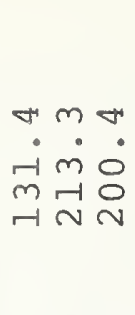 & 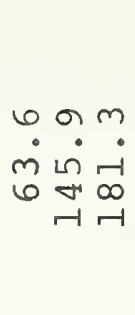 & 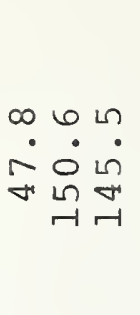 & 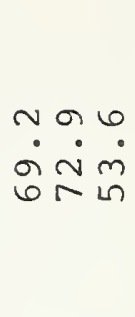 & $\begin{array}{ll}6 & 0 \\
\dot{0} & 0 \\
0 & 0\end{array}$ \\
\hline $\begin{array}{l}3 \\
0 \\
0 \\
0 \\
-1 \\
-\pi \\
+3 \\
5 \\
\sigma \\
0\end{array}$ & $\begin{array}{l}0 \\
0 \\
0 \\
0 \\
5 \\
4-1 \\
4-1 \\
-11 \\
01\end{array}$ & $\begin{array}{l}E \\
0 \\
1 \\
0\end{array}$ & 1 & $\begin{array}{l}\text { Mor } \\
m: \dot{m} \\
00\end{array}$ & 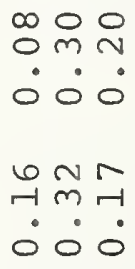 & 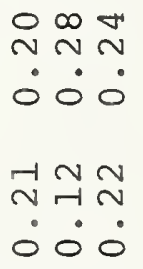 & 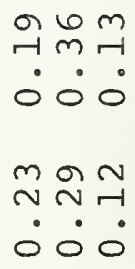 & 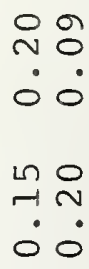 \\
\hline 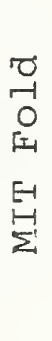 & 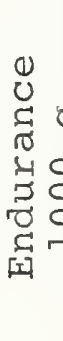 & $\begin{array}{c}0 \\
0 \\
0 \\
0 \\
0 \\
0 \\
01 \\
4-1 \\
0 \\
0 \\
0 \\
-1 \\
-10 \\
0 \\
0 \\
0\end{array}$ & 1 & 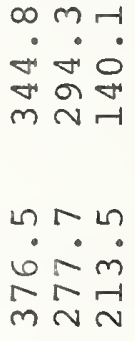 & 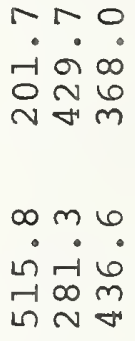 & 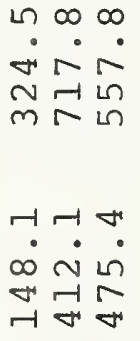 & 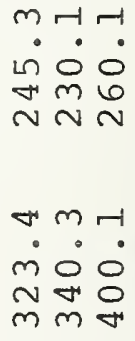 & 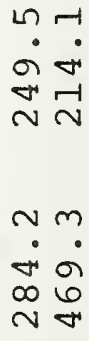 \\
\hline 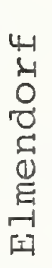 & $\begin{array}{l}4 \\
0 \\
0 \\
E-1\end{array}$ & or & 11 & 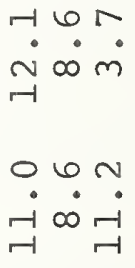 & $\begin{array}{l}r \infty \\
\sim \sim 0 \\
\sim \\
\sim\end{array}$ & 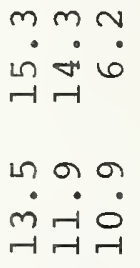 & 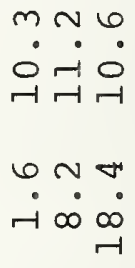 & 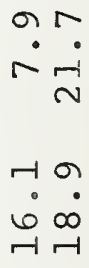 \\
\hline $\begin{array}{c}4-1 \\
0 \\
0 \\
0\end{array}$ & 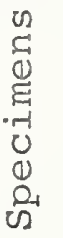 & & 1.7 & 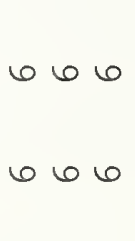 & $\begin{array}{l}666 \\
606\end{array}$ & 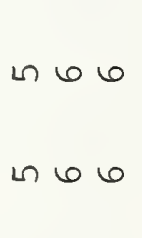 & 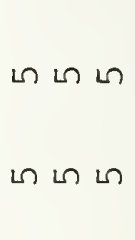 & $\begin{array}{l}6 \mathrm{~L} \\
6 \mathrm{~L}\end{array}$ \\
\hline & 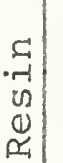 & 010 & & 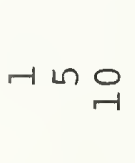 & 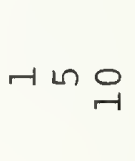 & r $\cap \underset{r}{0}$ & H $10 \underset{-1}{0}$ & $\begin{array}{c}4 \\
0 \\
0+ \\
0 \\
0 \\
\sigma \\
\sigma \\
3\end{array}$ \\
\hline & 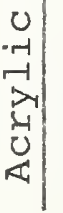 & $\begin{array}{l}0 \\
D_{1} \\
\text { E1 }\end{array}$ & & $\begin{array}{c}-1 \\
0 \\
1 \\
1\end{array}$ & $\begin{array}{c}a \\
m \\
1 \\
n\end{array}$ & $\begin{array}{c}0 \\
1 \\
0 \\
1 \\
1\end{array}$ & $\begin{array}{l}\overrightarrow{1} \\
1 \\
u\end{array}$ & \begin{tabular}{l}
0 \\
0 \\
0 \\
4 \\
+1 \\
\multicolumn{1}{c}{} \\
0 \\
0
\end{tabular} \\
\hline
\end{tabular}




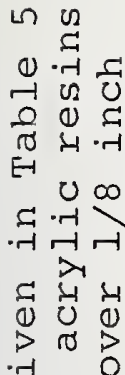

尚

ज 0

०.न ह

-

सा

is $>$

(1) 0

대용ㅇㅇ

त्र.तन

0,3

त

त) 0.7

$0+x$

० 010

>

त

U 1

$4+1$

400

I

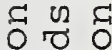

- 1 C

+1)

ه 도.

30

(1)

क्ष

त 상.

मे 4 is

त 0 तर

$\begin{array}{llll}0 & 0 & 0 & 0 \\ 1 & 3 & 0 & -1\end{array}$

त)

Ш

a

\begin{tabular}{|c|c|c|c|c|c|c|c|c|}
\hline 4 & 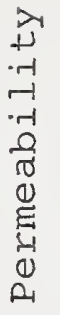 & $\begin{array}{l}\Xi \\
0 \\
U\end{array}$ & & 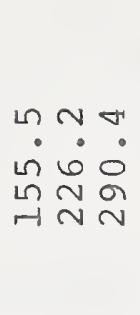 & $\begin{array}{l}0 \mathrm{~mm} \\
\dot{0} \dot{\mathrm{N}} \\
\mathrm{N}\end{array}$ & 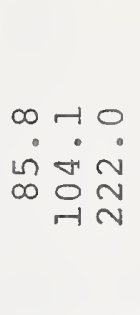 & 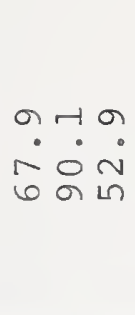 & $\begin{array}{l}\text { a } \\
\dot{m} \dot{ } \\
\dot{m}\end{array}$ \\
\hline 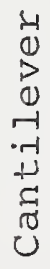 & $\begin{array}{l}0 \\
01 \\
0 \\
01 \\
4-1 \\
4-1 \\
01 \\
+1 \\
01\end{array}$ & $\begin{array}{l}5 \\
0 \\
1 \\
0\end{array}$ & 117 & $\begin{array}{lll}\pi & \infty & 0 \\
0 & 0 & 0 \\
0 & 0 & 0\end{array}$ & 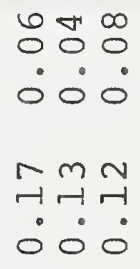 & 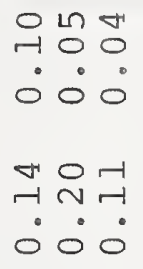 & 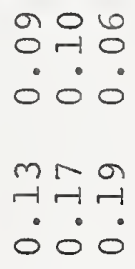 & 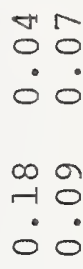 \\
\hline $\begin{array}{l}0 \\
01 \\
0 \\
14 \\
E-1 \\
{ }_{2}^{-1}\end{array}$ & 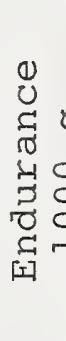 & $\begin{array}{c}n \\
0 \\
x \\
0 \\
0 \\
-1 \\
41 \\
0 \\
0 \\
-1 \\
-1 \\
3 \\
0 \\
0 \\
0\end{array}$ & $1-7$ & 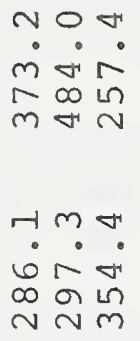 & 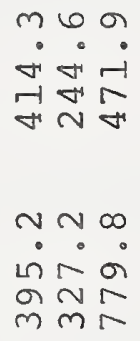 & 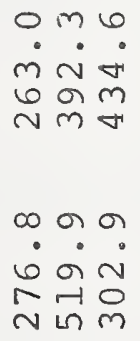 & 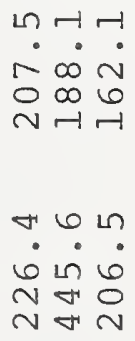 & 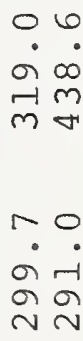 \\
\hline 年 & $\begin{array}{l}\frac{1}{\sigma} \\
0 \\
\text { E-1 }\end{array}$ & of & -7 & 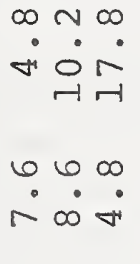 & 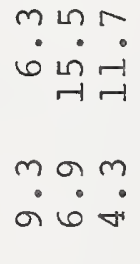 & 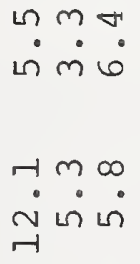 & 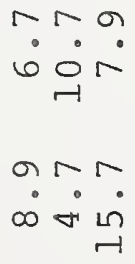 & 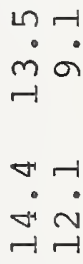 \\
\hline $\begin{array}{c}4-1 \\
0 \\
0 \\
0\end{array}$ & 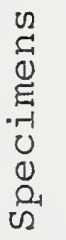 & & $1-7$ & 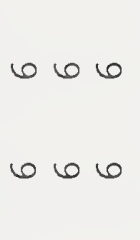 & 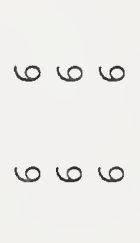 & 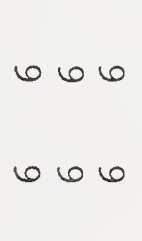 & $\begin{array}{l}\text { un un in } \\
\text { nin in }\end{array}$ & $\begin{array}{l}6 \text { in } \\
6 \text { n }\end{array}$ \\
\hline & 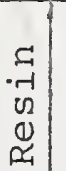 & olo & & r n & $\rightarrow$ 눔 & 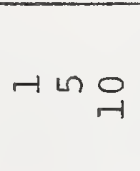 & $\begin{aligned} 10 \\
.\end{aligned}$ & $\begin{array}{cc}4 \\
0 \\
0 \\
0 \\
-4 \\
0 \\
0 \\
0\end{array}$ \\
\hline & 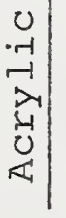 & (1) & & $\begin{array}{c}-1 \\
m \\
1 \\
1 \\
n-1\end{array}$ & $\begin{array}{l}a \\
m \\
1 \\
n\end{array}$ & $\begin{array}{c}0 \\
-1 \\
0 \\
1 \\
17\end{array}$ & $\begin{array}{c}-1 \\
0 \\
1 \\
u \\
1\end{array}$ & $\begin{array}{l}n \\
1 \\
0 \\
4 \\
1 \\
E \\
0 \\
0\end{array}$ \\
\hline
\end{tabular}




\begin{tabular}{|c|c|c|c|c|c|c|}
\hline 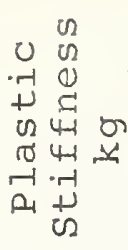 & $\mapsto$ & 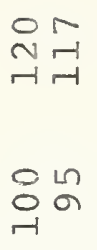 & 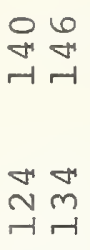 & 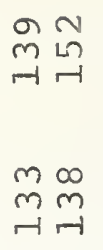 & 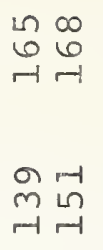 & 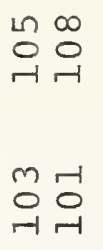 \\
\hline 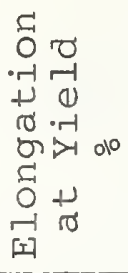 & $\mapsto$ & $\begin{array}{l}r r \\
00 \\
0 \\
00\end{array}$ & $\begin{array}{l}r i \\
00 \\
r \infty \\
0\end{array}$ & $\begin{array}{l}\infty r \\
0 \\
0 \\
0 \\
0\end{array}$ & $\begin{array}{l}0 . \\
0 \\
0 \\
0\end{array}$ & $\begin{array}{l}0 r \\
00 \\
0 R \\
00\end{array}$ \\
\hline 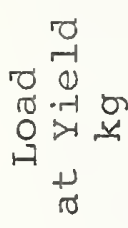 & 3 & 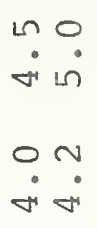 & 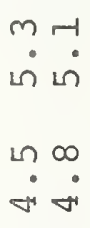 & 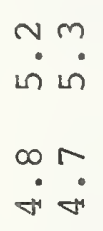 & 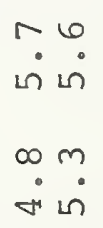 & 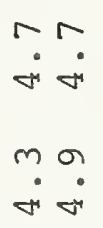 \\
\hline 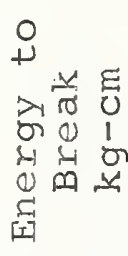 & $\mapsto$ & 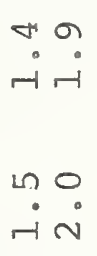 & $\begin{array}{l}o m \\
\dot{v} \dot{v} \\
-i v \\
\dot{v}\end{array}$ & $\begin{array}{l}O m \\
\dot{N} \dot{v} \\
\dot{\sim} \dot{n} \\
\dot{N}\end{array}$ & $\begin{array}{l}\dot{N} \\
\dot{0} \\
\dot{N} \\
\dot{N}\end{array}$ & 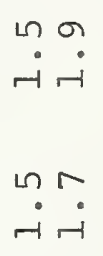 \\
\hline 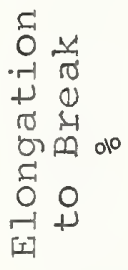 & 3 & $\begin{array}{l}\because \dot{n} \\
\dot{N} \dot{m} \\
\dot{n} \dot{m} \\
\dot{m} \dot{r}\end{array}$ & $\begin{array}{l}\forall \infty \\
\dot{m} \dot{m} \\
\infty m \\
\dot{m} \dot{m}\end{array}$ & $\begin{array}{l}n \dot{r} \\
\dot{m} \dot{m} \\
\infty \ddot{m} \\
\dot{m} \dot{m}\end{array}$ & 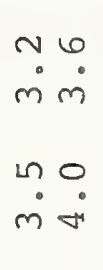 & $\begin{array}{l}06 \\
\dot{m} \dot{m} \\
\dot{r} \ddot{m} \\
\dot{m} \dot{m}\end{array}$ \\
\hline 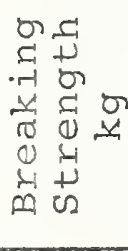 & 3 & $\begin{array}{l}0 \infty \\
0 \\
\text { in } \\
\text { in } \\
\text { in }\end{array}$ & 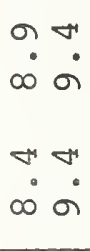 & $\begin{array}{l}\sigma \infty \\
\infty \dot{\sigma} \\
+\ddot{\sigma} \\
\infty \dot{0}\end{array}$ & 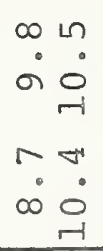 & $\begin{array}{l}-0 \\
r \infty \\
00 \\
0\end{array}$ \\
\hline 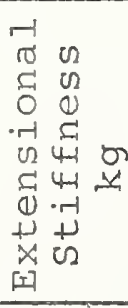 & $\mapsto$ & $\begin{array}{l}N \text { in } \\
\text { in }\end{array}$ & $\begin{array}{ll}m & m \\
i n & m\end{array}$ & $\begin{array}{l}6 \\
m \\
6\end{array}$ & $\begin{array}{ll}\varpi & \sigma \\
\infty & \sigma\end{array}$ & 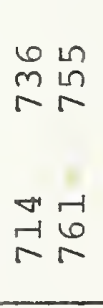 \\
\hline 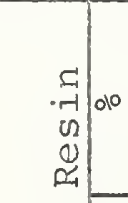 & & 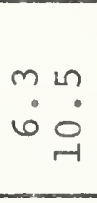 & $\vec{\sim} \dot{\sim}$ & $\begin{array}{l}\text { in } \\
\dot{0}\end{array}$ & 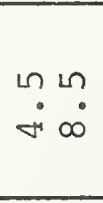 & $\begin{array}{ll}ت 4 & \\
4 & \\
0 & 0 \\
+ & 0 \\
\sigma & 0 \\
3 & 4\end{array}$ \\
\hline $\begin{array}{c}0 \\
-1 \\
-1 \\
2 \\
3 \\
0 \\
0 \\
\text { a }\end{array}$ & & $\begin{array}{l}\text { 더 } \\
0 \\
0 \\
1 \\
1\end{array}$ & $\begin{array}{l}a \\
m \\
m \\
1 \\
n\end{array}$ & $\begin{array}{l}0 \\
\qquad 1 \\
6 \\
1 \\
1\end{array}$ & 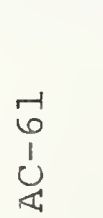 & $\begin{array}{l}0 \\
0-1 \\
0 \\
.1 \\
+1 \\
1 \\
0 \\
0\end{array}$ \\
\hline
\end{tabular}




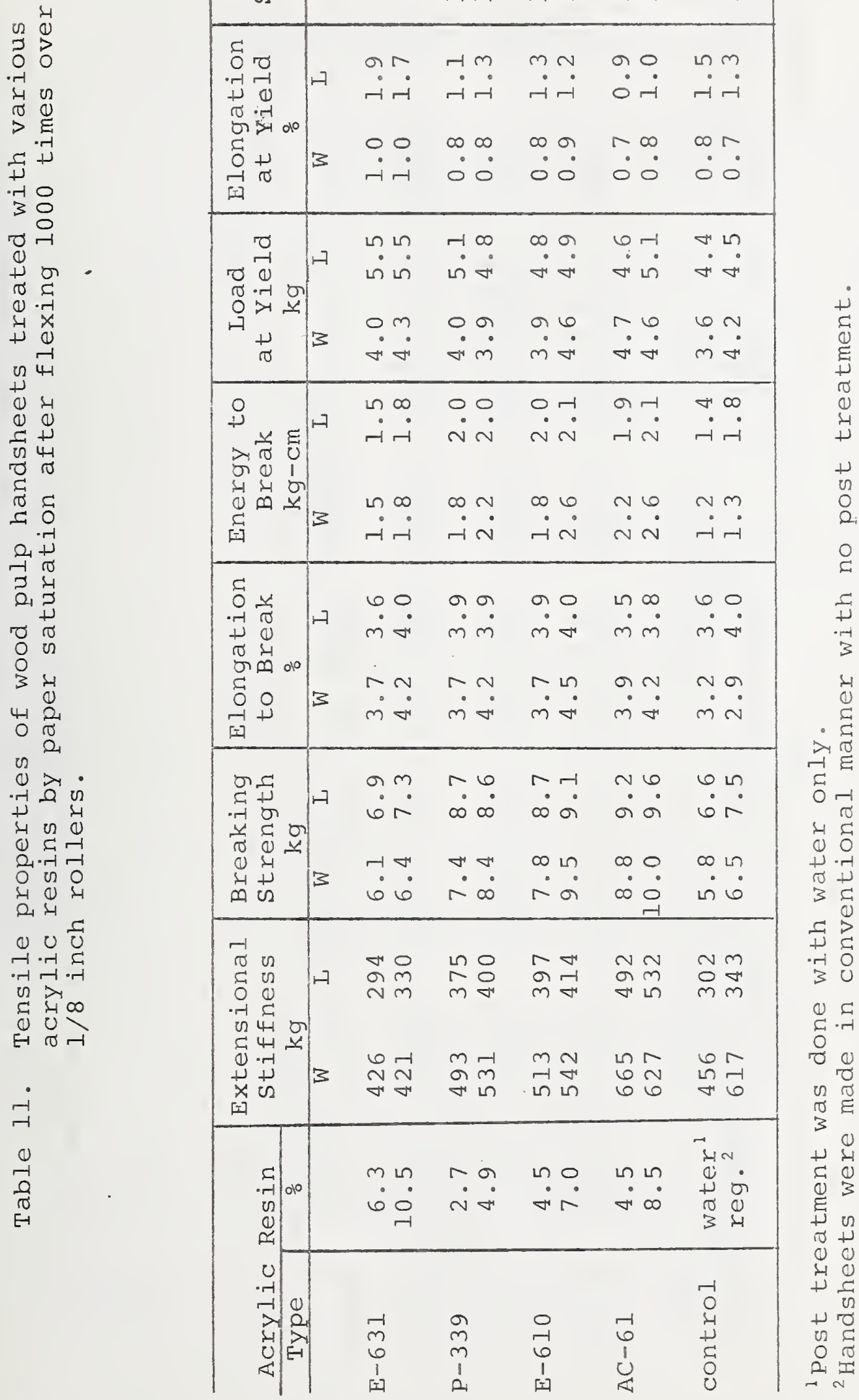




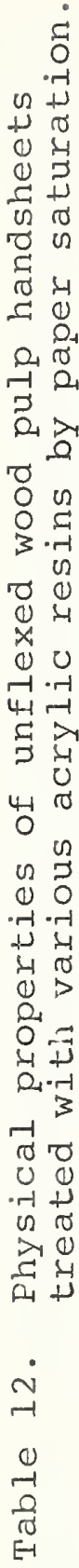

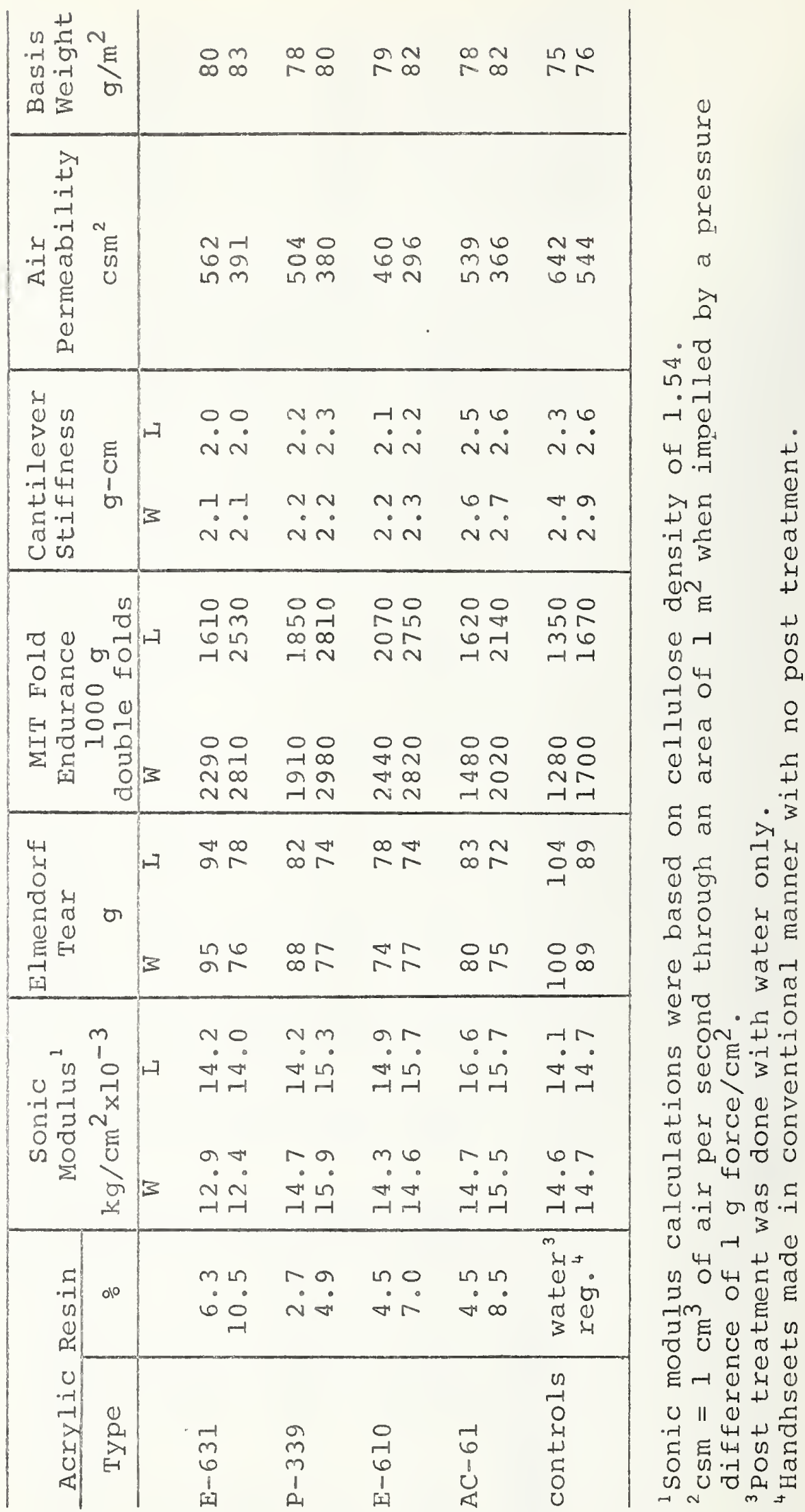




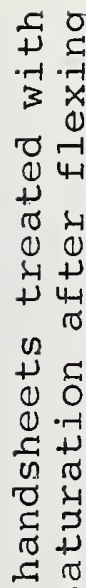

م 2

다낭

उ) 0

口,

\%

त्र क्षे

3 ล

4 $थ$ थ

O ⿷匚 .

os us $\infty$

(1) 0

if म्न

th 0 मr

$0 \cdot r 0$

겅

प्र त्र

0,0 थ

-1

U.

- $\rightarrow 0$

n. - 0

거엉

단 0

$\dot{m}$
-1
0
-1
$\tilde{\sigma}$
$\in$

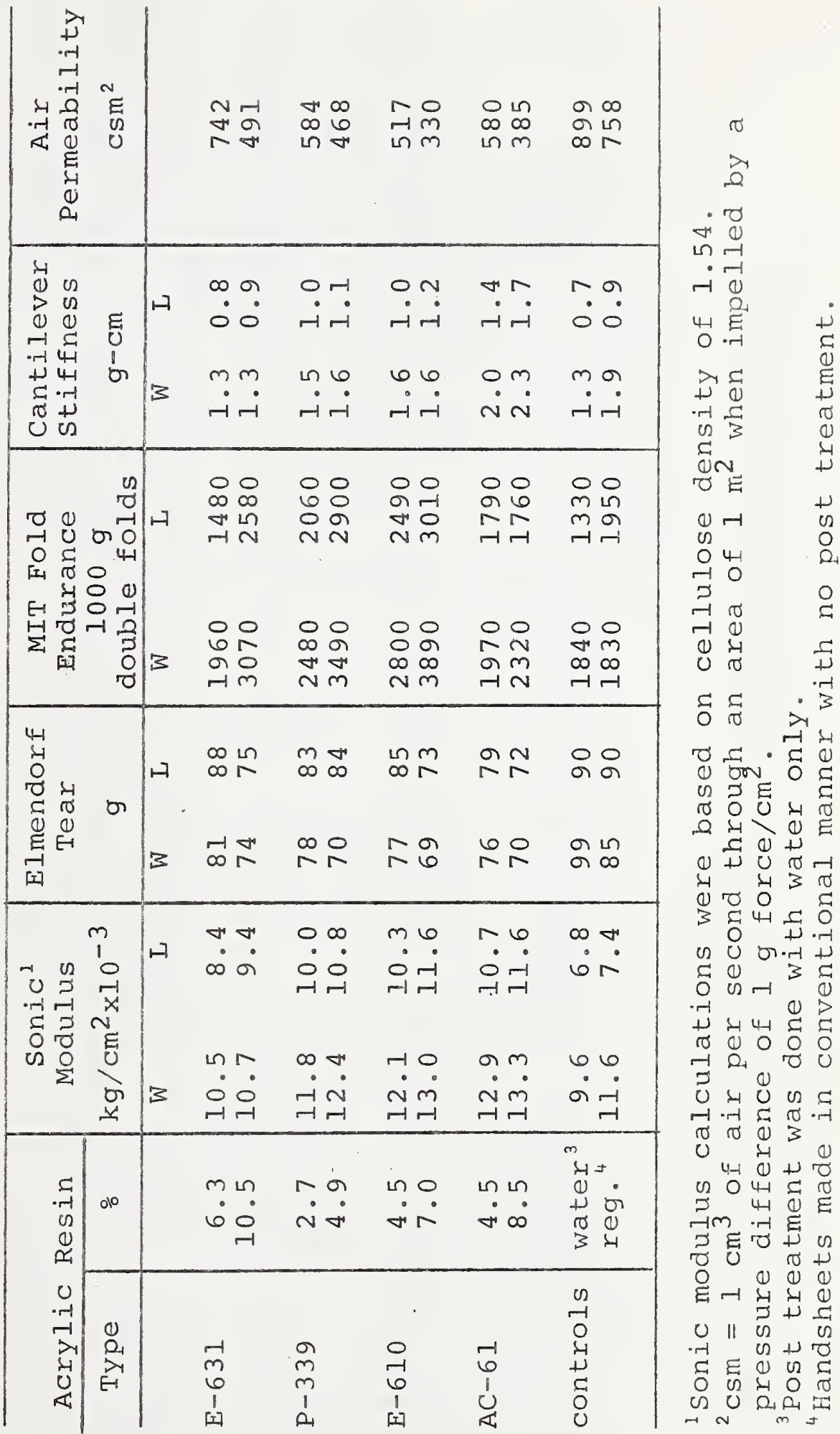




\begin{tabular}{|c|c|c|c|c|c|c|c|}
\hline $\begin{array}{l}0 \\
-1 \\
+1 \\
02 \\
0 \\
-1 . \\
-1\end{array}$ & 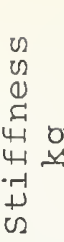 & $\mapsto$ & $\begin{array}{l}\sigma r \\
\dot{m} 0 \\
r \\
m \\
\dot{r}\end{array}$ & $\begin{array}{l}\sim m \\
\sim \dot{\infty} \\
+6 \\
\dot{\sim} \dot{\sigma}\end{array}$ & 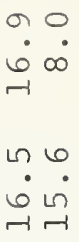 & $\begin{array}{l}\dot{r} \\
\dot{\sim} \\
\dot{r} \\
\sigma \\
\dot{\sim} \\
\dot{\sim}\end{array}$ & 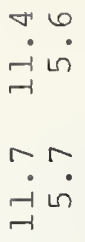 \\
\hline 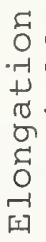 & $\begin{array}{l}\sigma \\
-1 \\
0 \\
-1 \\
>-1 \\
+\infty \\
+\infty\end{array}$ & 3 & $\begin{array}{ll}\infty & \text { Ln } \\
0 & 0 \\
0 & 0 \\
0 & 0 \\
1 & 0 \\
0 & 0 \\
0 & 0\end{array}$ & $\begin{array}{ll}7 & \infty \\
-1 & 0 \\
0 & 0 \\
0 & 0 \\
0 & 0 \\
0 & 0\end{array}$ & 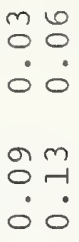 & 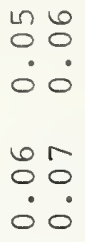 & $\begin{array}{ll}1 & 0 \\
0 & 0 \\
0 & 0 \\
0 & N \\
0 & -1 \\
0 & 0\end{array}$ \\
\hline $\begin{array}{l}0 \\
0 \\
0 \\
1\end{array}$ & 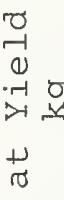 & 13 & 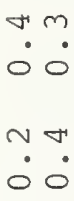 & 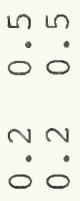 & $\begin{array}{l}\because \dot{0} \\
\dot{0} \\
\dot{0} \dot{0}\end{array}$ & $\begin{array}{l}m \overrightarrow{0} \\
\dot{0} \dot{0} \\
\dot{0} \dot{0}\end{array}$ & 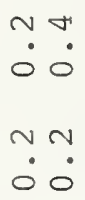 \\
\hline 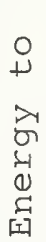 & 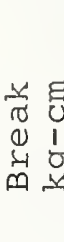 & 3 & 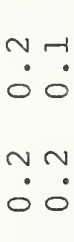 & $\begin{array}{l}\because m \\
\dot{0} 0 \\
-10 \\
00\end{array}$ & $\begin{array}{l}\text { m } \\
\dot{0} \\
\sim \text { N } \\
\dot{0}\end{array}$ & $\begin{array}{l}\dot{1} \\
\dot{0} \\
m \\
\dot{0}\end{array}$ & $\begin{array}{c}\text { N } \\
\dot{0} \\
\dot{0} \\
\dot{0} \\
\dot{0}\end{array}$ \\
\hline 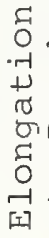 & $\begin{array}{l}\frac{x}{0} \\
0 \\
4 \\
\text { की } \\
+10 \\
+\end{array}$ & $1-1$ & $\begin{array}{l}\because v \\
\dot{0} \\
\text { m } \\
\dot{0}\end{array}$ & $\begin{array}{l}N \sim \\
\dot{O} 0 \\
N-1 \\
\dot{0} \dot{0}\end{array}$ & $\begin{array}{l}\because \mathrm{m} \\
0 \\
0 \\
\therefore \dot{0}\end{array}$ & $\begin{array}{l}\because m \\
\dot{0} 0 \\
m m \\
\dot{0} \dot{0}\end{array}$ & $\begin{array}{l}m-1 \\
\dot{0} \dot{0} \\
m+ \\
\dot{0} \dot{0}\end{array}$ \\
\hline 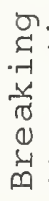 & 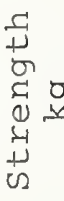 & $1+1$ & 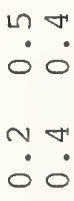 & $\begin{array}{l}\text { L } \\
\dot{0} 0 \\
\text { m L } \\
\dot{0} 0\end{array}$ & $\begin{array}{l}6 \% \\
0 \\
0 \\
0 \\
0\end{array}$ & $\begin{array}{l}m \text { m } \\
\dot{0} 0 \\
\dot{0} 0\end{array}$ & $\begin{array}{l}\text { ! } \\
\dot{\circ} 0 \\
0 \text { L } \\
\dot{0} 0\end{array}$ \\
\hline 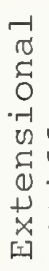 & 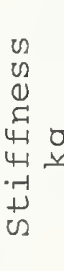 & 1 & 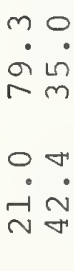 & 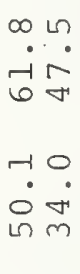 & 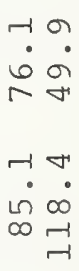 & 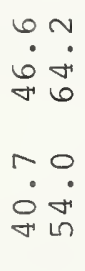 & 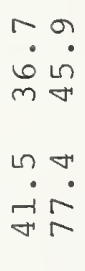 \\
\hline $\begin{array}{l}\text { 岁 } \\
\dot{0} \\
\dot{2}\end{array}$ & $\begin{array}{l}0 \\
己 \\
0 \\
0 \\
.1 \\
U \\
0 \\
0 . \\
0 \\
0\end{array}$ & $1+1$ & م & 66 & เ & ᄂ & 6 60 \\
\hline & 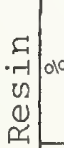 & & $\begin{array}{l}m \text { n } \\
\dot{0} 0 \\
\end{array}$ & $\begin{array}{l}\sim \\
\sim\end{array}$ & $\begin{array}{ll}\text { L } & 0 \\
\dot{\nabla} & 0\end{array}$ & : & 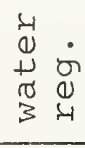 \\
\hline & $\begin{array}{ll}0 \\
\cdot-1 \\
-1 \\
-1 \\
0 \\
0 \\
0\end{array}$ & & $\begin{array}{l}-1 \\
m \\
0 \\
1 \\
\text { a1 }\end{array}$ & $\begin{array}{l}a \\
m \\
m \\
1 \\
n\end{array}$ & $\begin{array}{l}0 \\
-1 \\
6 \\
1 \\
1\end{array}$ & $\begin{array}{l}-1 \\
0 \\
1 \\
u\end{array}$ & $\begin{array}{l}n \\
-1 \\
0 \\
4 \\
4 \\
0 \\
0 \\
0\end{array}$ \\
\hline
\end{tabular}




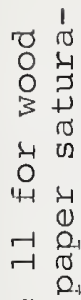

(1)

त) थ

\& 4

द.

U ज)

द 40

$>0$

तन

ठात्त

( 다. द्व

(1) U

-

it $v$.

(i) ว

아은 넌

$0 \cdot-40$

प्रत

(1) ज

ता है

U. $\rightarrow$

(1)

+ 舟 0

1
40
+10

0 西

(4)

대ㅇㅓㅕ

- -1 .

$\sigma+1)$

त (1)

$\rightarrow 04$

(1) 5

त 4

ठ

प1 8

त $5 \pi$

0

今 $\mathrm{O}_{4} \mathrm{C}$

+ $ح$.

w 0

in

0
-1
0
0

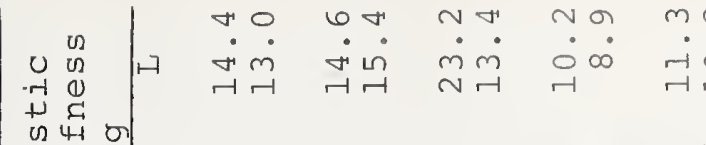

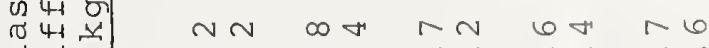

न्ل 3 in in

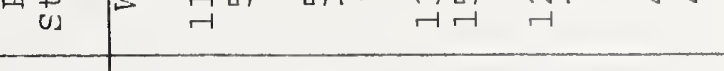

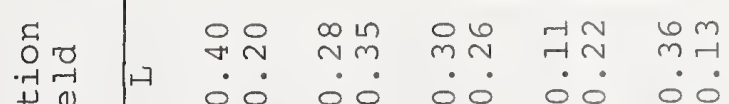

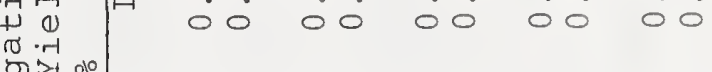

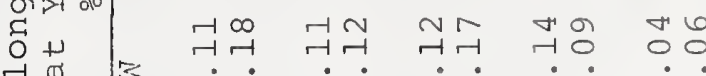

II

ก

ه

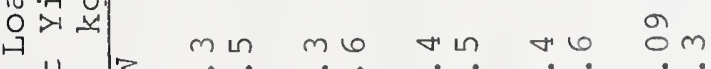

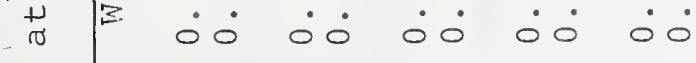

H

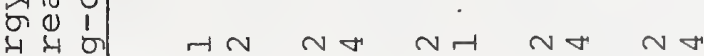

(1) 留 0000000000

g. $n m$ nn th nm ma

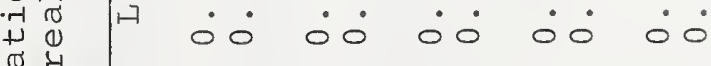

否

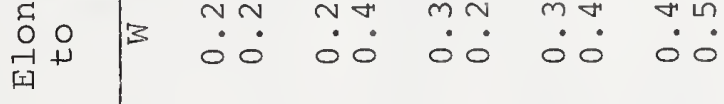

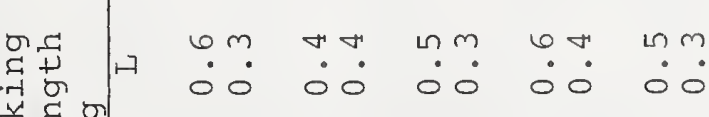

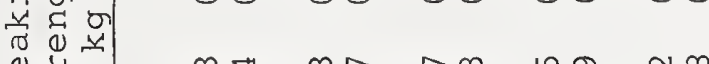

(1) 岑 5 ?

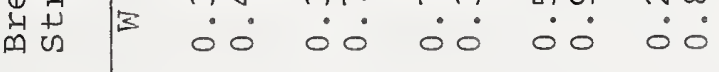

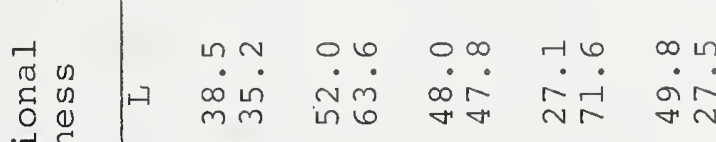

U. 41 o

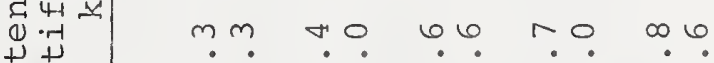

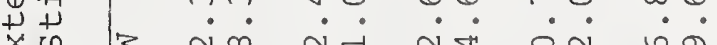

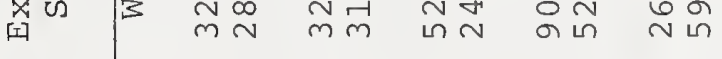

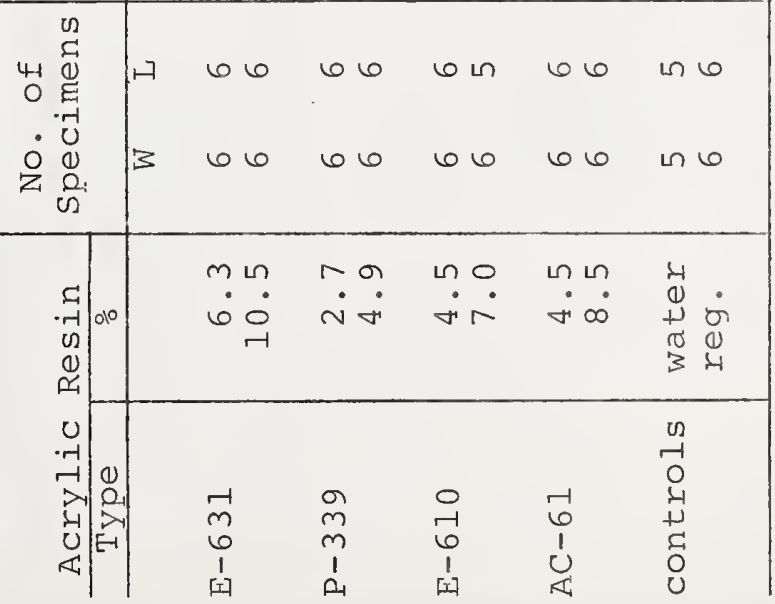




\begin{tabular}{|c|c|c|c|c|c|c|c|c|}
\hline 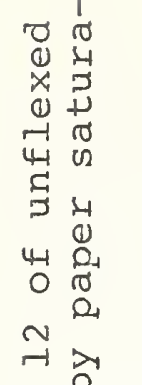 & & 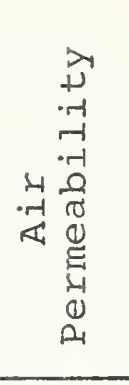 & $\begin{array}{l}E \\
0 \\
0\end{array}$ & $\begin{array}{l}r \quad b \\
\dot{m} \dot{m}\end{array}$ & $\begin{array}{l}m \\
\dot{\sim} \\
\vec{v}\end{array}$ & $\begin{array}{l}a \infty \\
\dot{\sim} \sim \\
m \sim\end{array}$ & $\begin{array}{l}\sigma b \\
\dot{m} \dot{\sim} \\
\dot{\sigma}\end{array}$ & $\begin{array}{l}0 \sim \\
\dot{0} \\
\dot{H}\end{array}$ \\
\hline 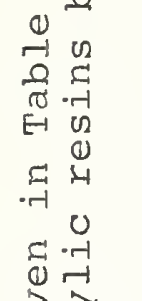 & & 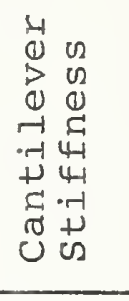 & $\begin{array}{l}\xi \\
0 \\
1 \\
0\end{array}$ & 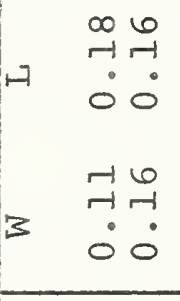 & $\begin{array}{ll}0 & 0 \\
-1 & -1 \\
0 & 0 \\
-1 & \infty \\
-1 & -1 \\
0 & 0\end{array}$ & 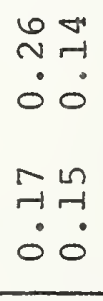 & $\begin{array}{l}\stackrel{0}{N} \\
\dot{0} \\
0 \\
N 0 \\
\sim 0 \\
0\end{array}$ & 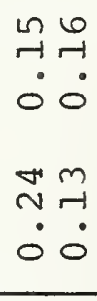 \\
\hline 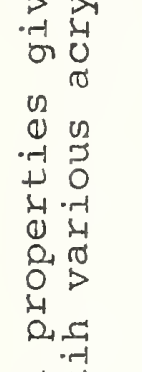 & & 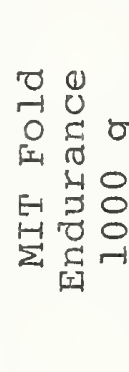 & $\begin{array}{c}0 \\
0 \\
x \\
0 \\
0 \\
4-1 \\
4 \\
0 \\
-1 \\
0 \\
3 \\
0 \\
0 \\
0\end{array} \mid$ & 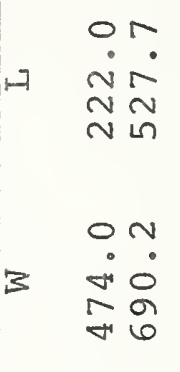 & $\begin{array}{ll}6 & 0 \\
\dot{0} & \dot{N} \\
\nabla & 0 \\
r & m \\
r & -1 \\
\dot{\infty} & 0 \\
\infty & \infty \\
\sim & \sigma\end{array}$ & 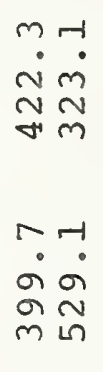 & 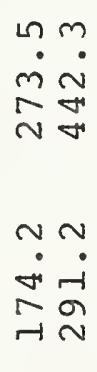 & 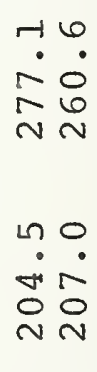 \\
\hline 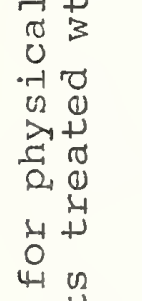 & & 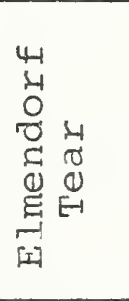 & or & 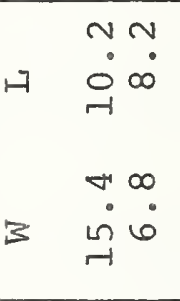 & 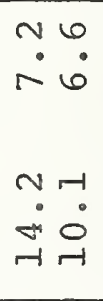 & $\begin{array}{l}0 m \\
\dot{r} \\
\dot{r} \\
\infty \\
\dot{\sim} \\
\dot{0}\end{array}$ & $\begin{array}{l}\forall \infty \\
\dot{\sigma} \dot{0} \\
\dot{0} 0 \\
\dot{m} \infty\end{array}$ & 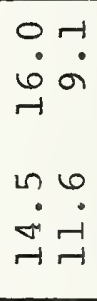 \\
\hline 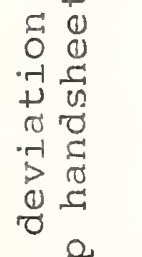 & & 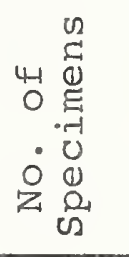 & & $\begin{array}{l}\text { ص } 6 \\
66\end{array}$ & $\begin{array}{l}66 \\
66\end{array}$ & $\begin{array}{l}66 \\
66\end{array}$ & $\begin{array}{l}60 \\
66\end{array}$ & $\begin{array}{l}60 \\
60\end{array}$ \\
\hline 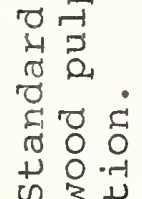 & & $\begin{array}{c}c \\
.-1 \\
0 \\
0 \\
21\end{array}$ & old & $\begin{array}{l}m \text { n } \\
0 \\
0 \\
0 \\
0\end{array}$ & $\begin{array}{l}r a \\
\dot{v} \dot{r}\end{array}$ & $\begin{array}{l}n 0 \\
\dot{0}\end{array}$ & $\begin{array}{l}n \text { In } \\
\dot{\sim} \infty\end{array}$ & $\begin{array}{ll}4 & 1 \\
0 & 0 \\
+ & 0 \\
0 & 0 \\
3 & 4\end{array}$ \\
\hline $\begin{array}{l}0 \\
0 \\
-1 \\
0 \\
\overrightarrow{0} \\
\overrightarrow{0}\end{array}$ & & \begin{tabular}{c}
0 \\
$0-1$ \\
-1 \\
\multirow{2}{4}{} \\
0 \\
0 \\
4
\end{tabular} & $\begin{array}{l}0 \\
2 \\
\text { E-1 }\end{array}$ & 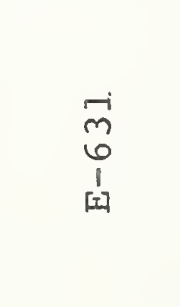 & $\begin{array}{l}a \\
m \\
m \\
1 \\
m\end{array}$ & $\begin{array}{l}0 \\
-1 \\
6 \\
1 \\
1 \\
\text { a }\end{array}$ & $\begin{array}{l}-1 \\
\emptyset \\
1 \\
u \\
4\end{array}$ & 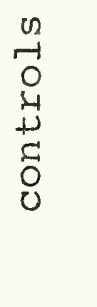 \\
\hline
\end{tabular}




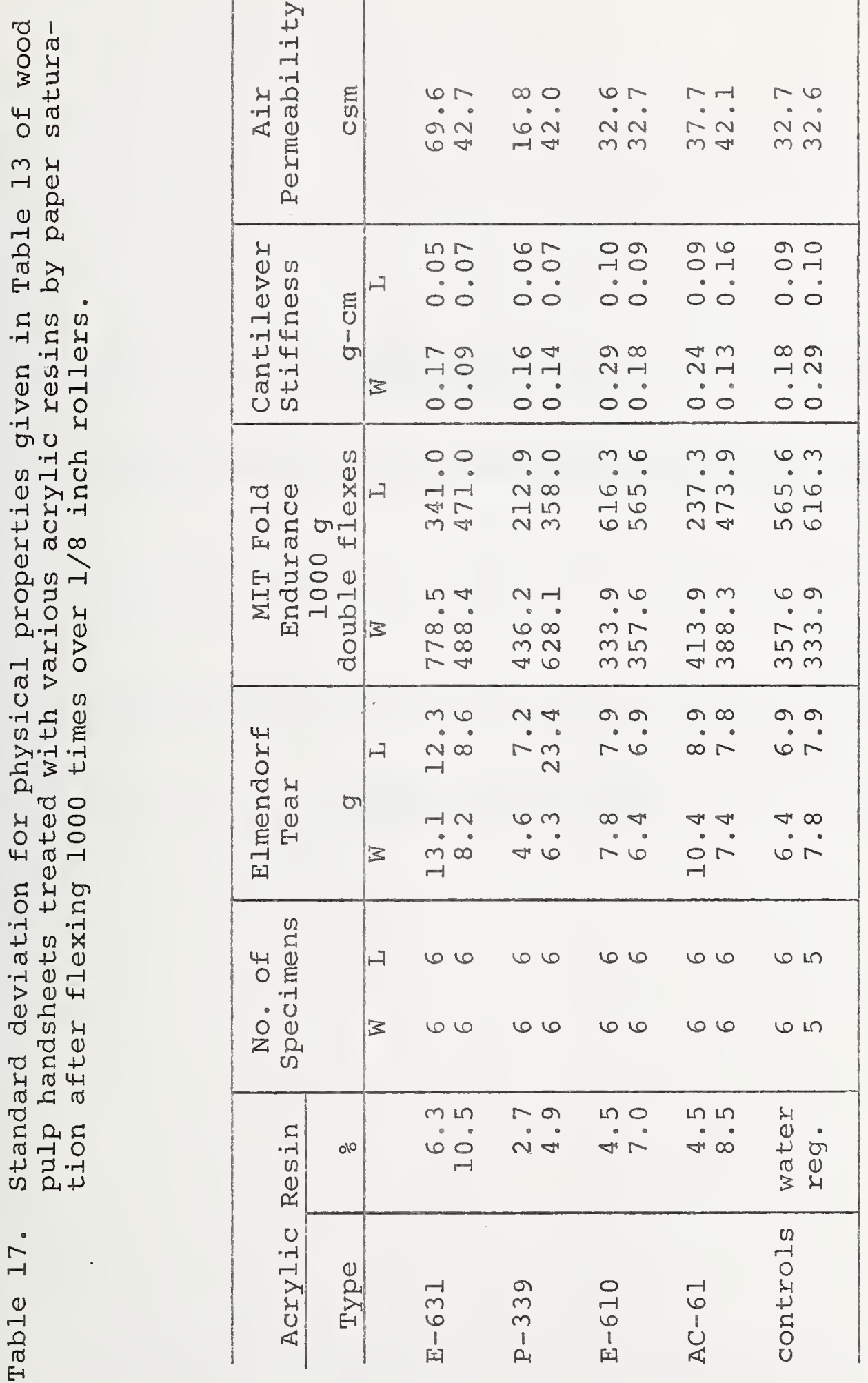





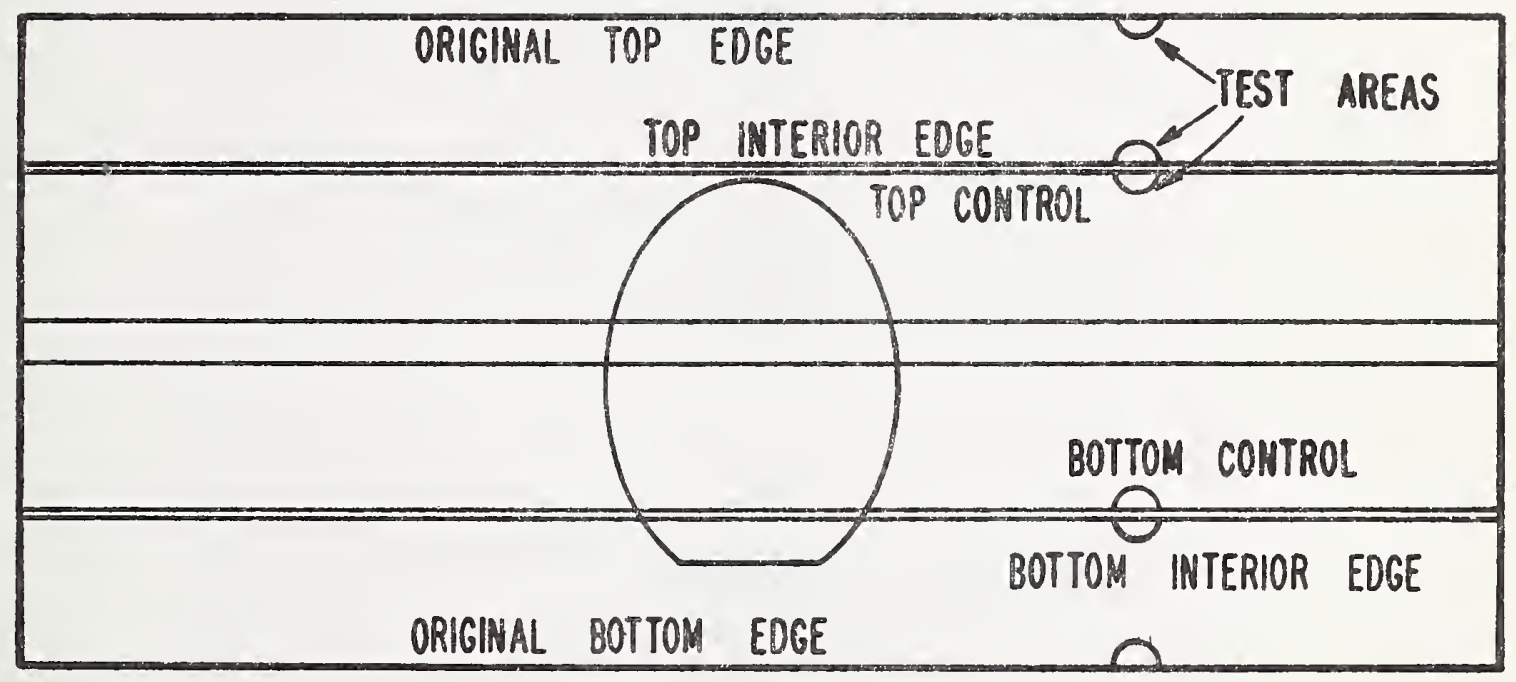

Figure 1. Specimen layout for redeemed currency for edge tear specimens. 



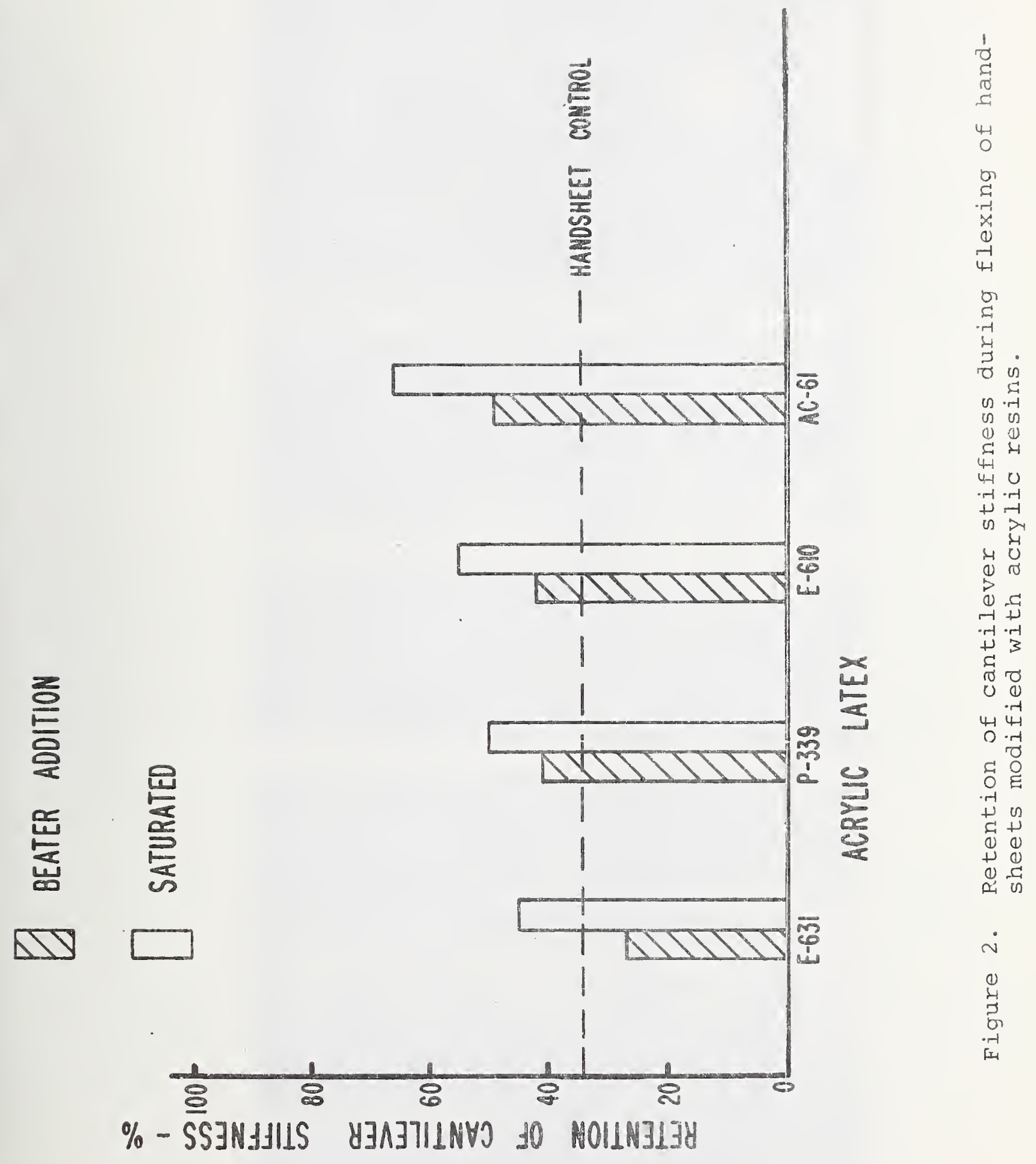




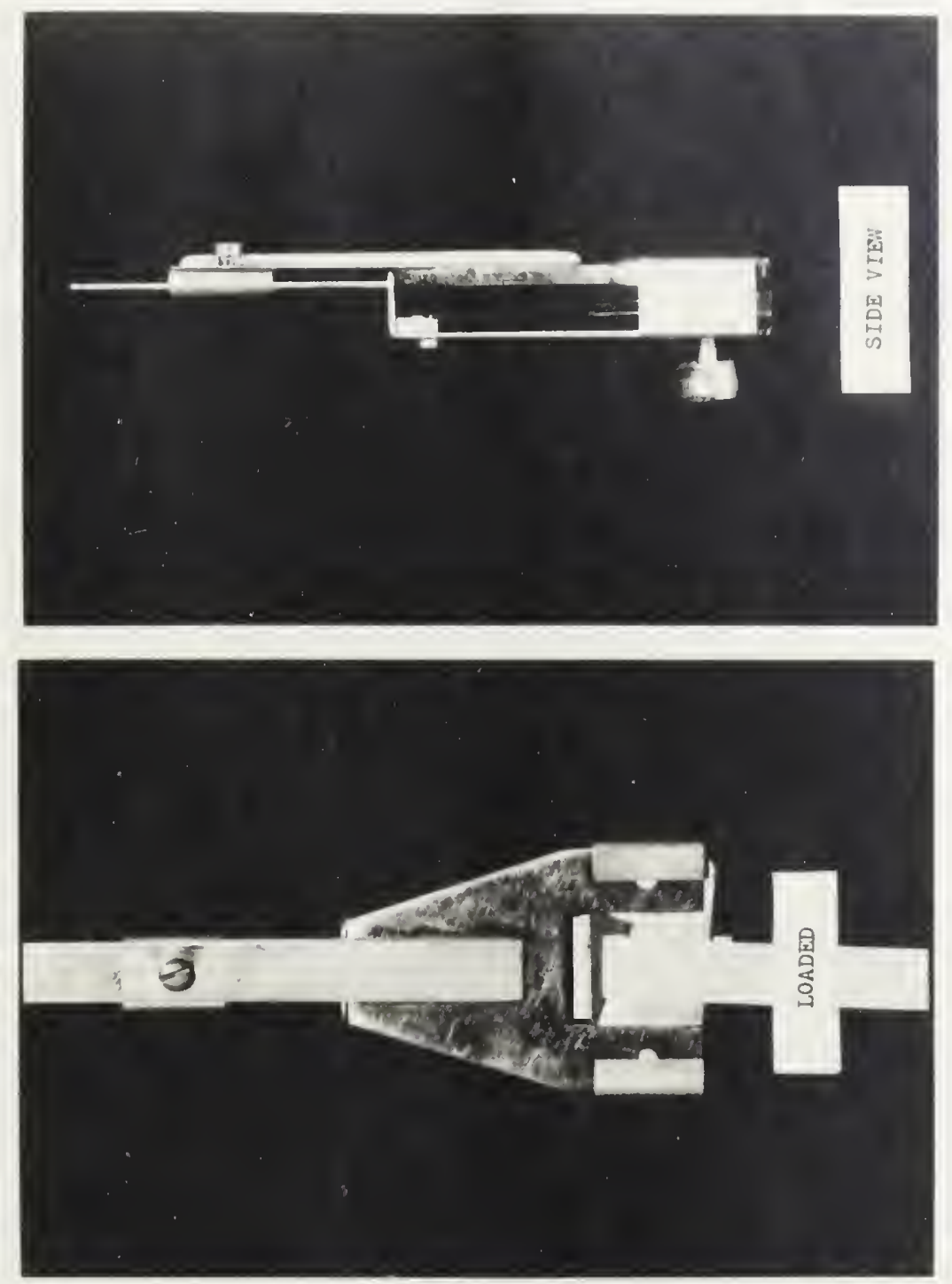

I

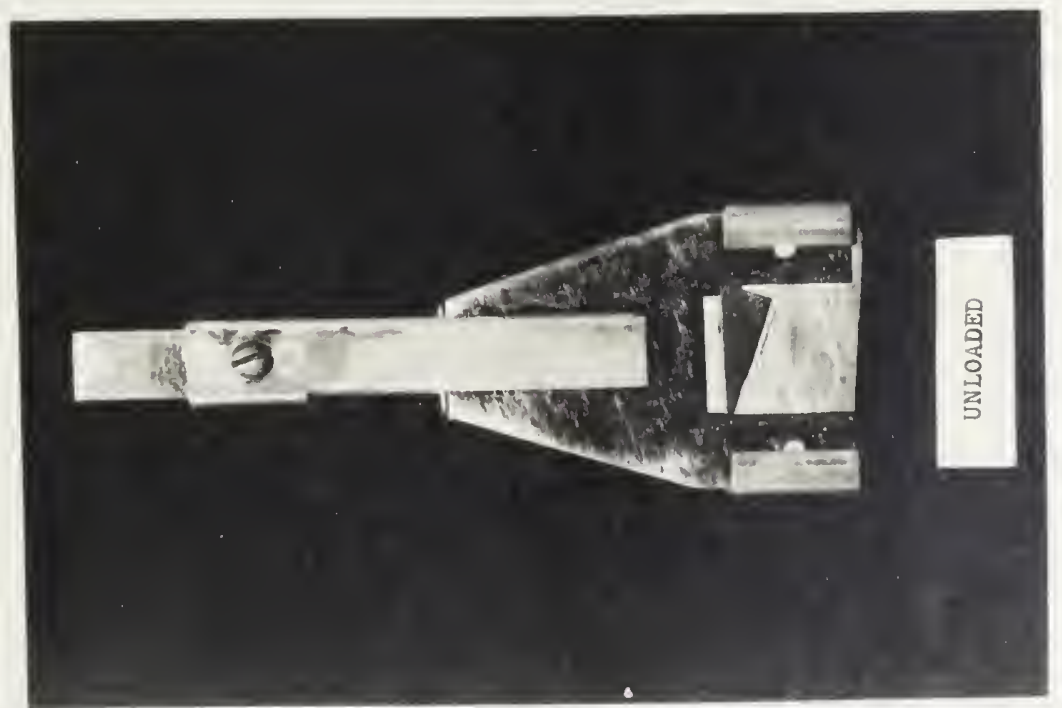



FORM NBS-114A (1-71)

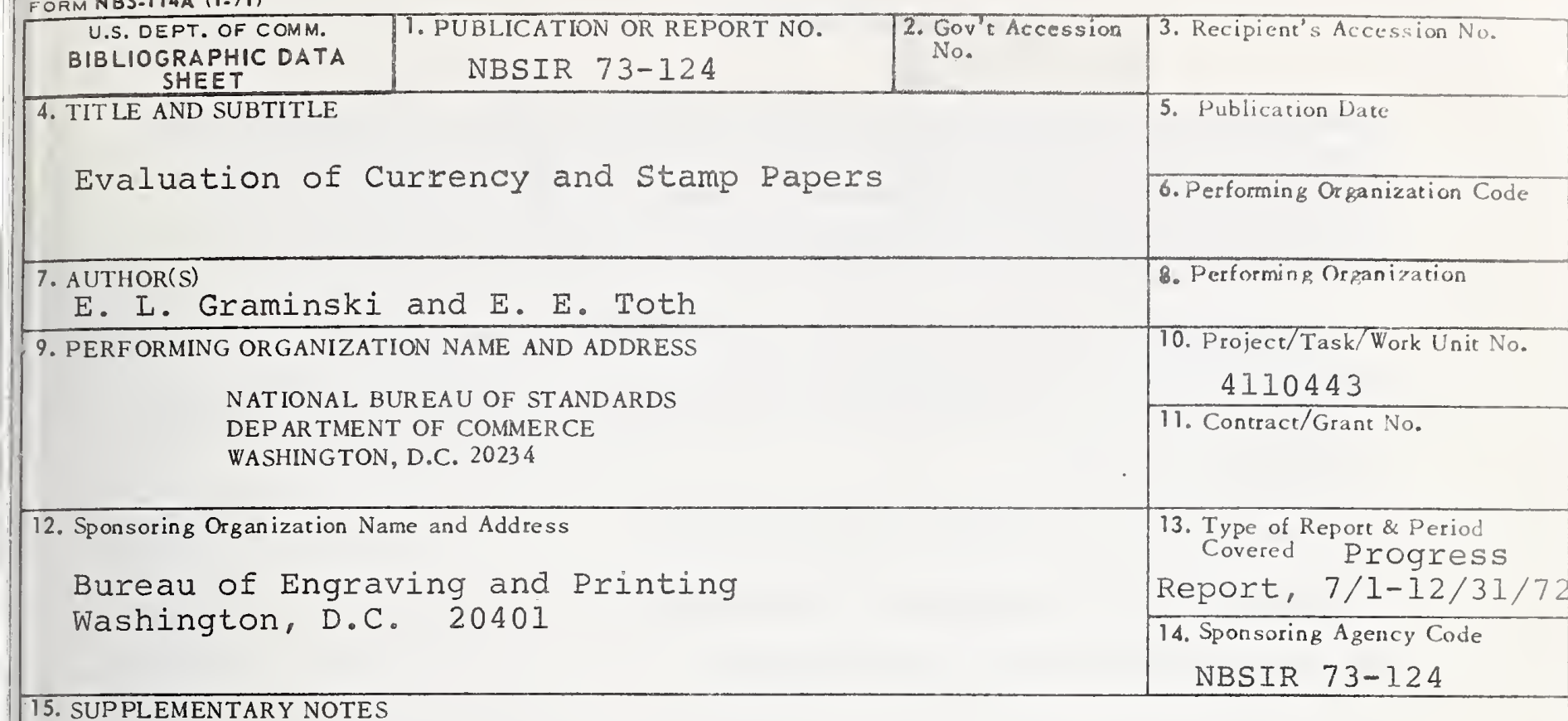

15. SUP PLEMENTARY NOTES

16. ABSTRACT (A 200-word or less factual summary of most significant information. If document includes a significant bibliography or literature survey, mention it here.)

As part of a continuing study for the Bureau of Engraving and Printing of the U.S. Department of Treasury, the disparity in the edge tear of flexed currency paper and redeemed currency and the possibility of improving the stiffness retention of paper by treatment with acrylic resins was studied. It was shown that the increase in edge tear of currency during circulation is caused by wear and is not due to the flexing a note receives during circulation. Also, paper can be modified with acrylic latexes by a paper saturation technique with the result that the paper has a marker. improvement in stiffness retention with flexing.

17. KEY WORDS (Alphabetical order, separated by semicolons)

Acrylic latexes; edge tear; paper; paper durability; stiffness retention.

18. AVAILABILITY STATEMENT

UNLIMIT ED.

XXFOR OFFICIAL DISTRIBUTION. DO NOT RELEASE TO NTIS.

\begin{tabular}{|l|l|}
$\begin{array}{l}\text { 19. SECURITY CLASS } \\
\text { (THIS REPORT) }\end{array}$ & 21. NO. OF PAGES \\
UNCL ASSIFIED & \\
\hline $\begin{array}{l}\text { 20. SECURITY CLASS } \\
\text { (THIS PAGE) } \\
\text { UNCL ASSIFIED }\end{array}$ & 22. Price \\
\hline
\end{tabular}


FORM NBS-114A: BIBLIOGRA PHIC DATA SHEET (REVERSE SIDE). This Bibiographic Data Sheet is an NBS adaptation of the form prescribed by COSATI guidelines (Appendix F. NBS Manual for Scientific and Technical Communications). Please complete with extreme care. This sheet will provide the basis for the literature citation of the publication, and in most cases it will become an integral part of the final publication itself.

A. Complete item 1 if information is available; otherwise, OTIP will complete later. (See K below.)

B. Ignore toned items 2,6, 8 , and 14; these are reserved for possible future use. Also, ignore item 3.

C. Complete items 4 and 7 .

D. Leave items 5, 21, and 22 blank; OTIP will complete.

E. Items 9, 19, and 20 are preprinted; you need add nothing.

F. Complete items 10,11, and/or 12 when applicable.

G. For item 13, enter "Final" or "Interim" and calendar period covered, as appropriate.

H. For item 15, enter other relevant information. (For example, upon receipt of completed Form NBS-266 from author, OTIP will enter the complete citation for NBS - authored papers published in non-NBS media.)

1. Complere items 16 and 17. Guidance is given in Secrion 4 and.Appendix B of the NBS Manual for Scientific and Technical Communications.

J. For item 18, enter one of the following:

"Unlimited" - for open-literature documents cleared under NBS editorial procedures, or

"For official distribution. Do not release to NTIS" - for limited, restricted, or need-to-know material. (Other availability statements are being developed. If the above are not applicable, contact OTIP for guidance.)

K. In completing item 1, use the brief designators shown in the right-hand column below. Each designator will be followed by the specific publication number for that item. This number will be the same in both the longer and briefer designators for the same document. For example: NBS Technical Note 548 will be equivalent to NBS-TN-548. You would enter NBS-TN-548 in item 1 of Form NBS-114A.

\section{NBS Identification}

NBS Technical Note

NBS Monograph

NBS Handbook

NBS Special Publication

NBS Applied Mathematics Series

NBS National Standard Reference Data Series

NBS Building Science Series

NBS Federal Information Processing Standards Publication

NBS Voluntary Product Standards

NBS Consumer Information Series

NBS Joumal of Research Section A

NBS Journal of Research Section B

NBS Journal of Research Section C

NBS Technical News Bulletin
NBS-TN-
NBS-MN-
NBS-HB-
NBS-SP-
NBS-AMS-
NBS-NSRDS-
NBS-BSS
NBS-FIPS-
NBS-PS-
NBS-CIS-
NBS-JRA-
NBS-JRB-
NBS-JRC-
NBS-TNB-

Since each paper in the three-volume NBS Journal of Research is assigned a specific designator, OTIP will add that designator to the appropriate Journal designator shown in the above right-hand column to obtain the entry for item 1 . 

